\title{
A role for TNF- $\alpha$ in alveolar macrophage damage-associated molecular pattern release
}

\author{
Morgan K. Collins, ${ }^{1}$ Abigail M. Shotland, ${ }^{1}$ Morgan F. Wade, ${ }^{1}$ Shaikh M. Atif, ${ }^{1}$ Denay K. Richards, ${ }^{2}$ \\ Manolo Torres-Llompart, ${ }^{2}$ Douglas G. Mack, ${ }^{1}$ Allison K. Martin, ${ }^{1}$ Andrew P. Fontenot, ${ }^{1,3}$ \\ and Amy S. McKee ${ }^{1,3}$ \\ 'Division of Allergy, Asthma and Clinical Immunology, Department of Medicine, ${ }^{2}$ Webb-Waring/Colorado Undergraduate \\ Summer Research Program, and ${ }^{3}$ Department of Immunology and Microbiology, University of Colorado School of Medicine, \\ University of Colorado Anschutz Medical Campus, Aurora, Colorado, USA.
}

\begin{abstract}
Chronic beryllium disease (CBD) is a metal hypersensitivity/autoimmune disease in which damageassociated molecular patterns (DAMPs) promote a break in $\mathrm{T}$ cell tolerance and expansion of $\mathrm{Be}^{2+} /$ self-peptide-reactive CD4 ${ }^{+}$T cells. In this study, we investigated the mechanism of cell death induced by beryllium particles in alveolar macrophages (AMs) and its impact on DAMP release. We found that phagocytosis of Be led to AM cell death independent of caspase, receptor-interacting protein kinases 1 and 3, or ROS activity. Before cell death, Be-exposed AMs secreted TNF- $\alpha$ that boosted intracellular stores of IL-1 $\alpha$ followed by caspase-8-dependent fragmentation of DNA. IL-1 $\alpha$ and nucleosomal DNA were subsequently released from AMs upon loss of plasma membrane integrity. In contrast, necrotic AMs released only unfragmented DNA and necroptotic AMs released only IL-1 $\alpha$. In mice exposed to Be, TNF- $\alpha$ promoted release of DAMPs and was required for the mobilization of immunogenic DCs, the expansion of Be-reactive $C D 4^{+} \mathrm{T}$ cells, and pulmonary inflammation in a mouse model of CBD. Thus, early autocrine effects of particle-induced TNF- $\alpha$ on AMs led to a break in peripheral tolerance. This potentially novel mechanism may underlie the known relationship between fine particle inhalation, TNF- $\alpha$, and loss of peripheral tolerance in T cell-mediated autoimmune disease and hypersensitivities.
\end{abstract}

Authorship note: MKC and AMS contributed equally.

Conflict of interest: The authors have declared that no conflict of interest exists.

Copyright: (c) 2020, American Society for Clinical Investigation.

Submitted: October 17, 2019

Accepted: April 1, 2020

Published: May 7, 2020.

Reference information: JCI Insight. 2020;5(9):e134356.

https://doi.org/10.1172/jci.

insight.134356.

\section{Introduction}

Resident alveolar macrophages (AMs) exposed to a variety of particles can initiate inflammation via the release of damage-associated molecular patterns (DAMPs) $(1,2)$. Sterile particles with crystalline morphology (e.g., metal hydroxide salts, silica) promote cell stress and in some cases cell death in macrophages (1-6). These factors drive lung inflammation and promote upregulation of costimulatory molecules on pulmonary conventional dendritic cells (cDCs) and thus regulate adaptive immune responses (1, $5,7-11)$. The release of IL-1 $\alpha$ from dying AMs following crystalline particle inhalation is unique because other macrophage populations do not release IL-1 $\alpha$ without a priming TLR signal $(1,12)$. Inhalation of fine particles can drive acute pulmonary inflammation, enhance adaptive immune responses to bystander or self-antigens, and increase the risk for developing allergy and certain types of autoimmune disease (13-23). Chronic beryllium disease (CBD) is both a metal-induced hypersensitivity and autoimmune disease to self-peptides (24). Exposure of AMs to crystalline forms of beryllium promotes their death and release of IL- $1 \alpha$ and DNA. These DAMPs drive accelerated migration of immunogenic cDCs from the lung to the lung-draining lymph nodes (LDLNs). Here, they can present a subset of self-peptides on $\mathrm{Be}^{2+}$-modified HLA-DP2 molecules to naive $\mathrm{CD} 4^{+} \mathrm{T}$ cells in a manner that breaks peripheral tolerance $(2$, $12,24,25)$. Once activated, $\mathrm{Be}^{2+} / \mathrm{HLA}-\mathrm{DP} 2-$ presented self-peptide-reactive ( $\mathrm{Be}^{2+} /$ self-peptide-reactive) $\mathrm{CD}^{+} \mathrm{T}$ cells differentiate into IFN- $\gamma$-secreting $\mathrm{CD}^{+}$effector $\mathrm{T}\left(\mathrm{T}_{\text {eff }}\right)$ cells that are licensed to enter the lung tissue $(2,12,26-28)$. There, they respond to $\mathrm{Be}^{2+} /$ self-peptides presented by antigen-presenting cells and orchestrate chronic pulmonary inflammation and progressive fibrosis. Thus, while many types of particles can promote pulmonary disease via the activation of the innate immune system alone, others, such as beryllium, can also promote development of $\mathrm{T}$ cell-mediated autoimmunity and hypersensitivity. 
Peripheral tolerance is controlled by steady-state cDCs (29-31); however, these cells become immunogenic in response to DAMPs. DAMPs engage innate damage recognition receptors, leading to the increased expression of costimulatory molecules on $\mathrm{CDC}$ membranes. The high expression of these molecules on immunogenic cDCs provides survival signals to naive $\mathrm{T}$ cells responding to the presented antigen (32-35). We have shown that AM cell death and DAMP release occur following single low-dose exposures to either beryllium sulfate (which forms beryllium hydroxide in airway fluids) or crystalline beryllium hydroxide (Be) particles (2). DNA and IL-1 $\alpha$ released from Be-exposed AMs induce upregulation of CD80 on cDCs via the MyD88-dependent receptors TLR9 and IL-1R1 $(2,12,36)$. In addition, DNA released by Be-exposed AMs promotes accelerated migration of cDCs from the lung to the LDLNs via TLR9 (2). These cDCs promote expansion and survival of effector and memory $\mathrm{CD} 4^{+} \mathrm{T}$ cells to presented bystander antigens $(2,12)$.

In this study, we found that Be-induced AM cell death requires phagocytosis and lysosomal destabilization but is independent of ROS and of caspase, cathepsin, receptor-interacting protein kinase 1 (RIP1K), or RIP3K activity. However, before cell death, TNF- $\alpha$ was released by AMs that had engulfed Be, leading to a caspase-independent increase in stores of intracellular IL-1 $\alpha$. This was followed by activation of caspase- 8 and caspase- 3 and fragmentation of DNA into nucleosomes. These immunogenic DAMPs were subsequently released by AMs upon loss of plasma membrane integrity. Systemic blockade of TNF- $\alpha$ or TNF- $\alpha$ deficiency in Be-exposed mice prevented the release of IL- $1 \alpha$ and DNA into the airways and impaired the previously reported effects of these DAMPs on inflammation and accumulation of CD80 ${ }^{\text {hi }}$ cDCs in the LDLNs $(2,12)$. We tested the role that these early innate pathways play in a mouse model of CBD using HLA-DP2-transgenic mice (DP2 mice) (26). These mice express both chains of the human HLA-DP2 molecule under control of the MHCII promoter, leading to surface expression of HLA-DP2 on DCs, B cells, macrophages, and thymic epithelial cells. In contrast to nontransgenic mice, DP2 mice exposed to Be generate a Be ${ }^{2+} /$ self-peptide-reactive $\mathrm{CD}^{+} \mathrm{T}_{\text {eff }}$ cell response (26). Similar to $\mathrm{CBD}$, these $\mathrm{Be}^{2+} /$ self-peptide-reactive $\mathrm{CD} 4{ }^{+} \mathrm{T}$ cells (and thus HLA-DP2) are required for mice to develop pulmonary immunopathology following low-dose exposure to Be. Using the DP2 model, we confirmed that cDCs and MyD88-dependent signaling were required for $\mathrm{Be}^{2+} /$ self-peptide-specific $\mathrm{CD}^{+} \mathrm{T}_{\text {eff }}$ cell responses in Beexposed DP2 mice. Finally, we found that TNF- $\alpha$ blockade in Be-exposed DP2 mice maintained peripheral $\mathrm{T}$ cell tolerance to $\mathrm{Be}^{2+} /$ self-peptide and prevented the development of immunopathology in the lung. This study is the first to our knowledge to show that particle-induced TNF- $\alpha$ secretion by AMs primes them to release high levels of immunogenic DAMPs into the airways upon particle-induced cell death and, thus, promotes a break in peripheral tolerance by mobilizing immunogenic cDCs to the LDLNs. This is a novel mechanism that may underlie known associations between TNF- $\alpha$, particle inhalation, and the development of $\mathrm{T}$ cell-mediated autoimmunity and hypersensitivity.

\section{Results}

Be particle-exposed AMs release both $I L-1 \alpha$ and DNA, in contrast to AMs undergoing apoptosis, necroptosis, or primary necrosis. AMs exposed to crystalline Be particles undergo cell death and release of IL-1 $\alpha$ and DNA within 18 hours of exposure. DNA and IL-1 $\alpha$ released from these cells act as DAMPs that promote recruitment of neutrophils and mobilization of immunogenic cDCs $(2,12)$. Different types of programmed cell death can result in differing profiles of DAMP release $(1,37)$. To define whether different types of programmed cell death in AMs led to release of IL-1 $\alpha$, DNA, or both, we quantified IL-1 $\alpha$ and DNA released from apoptotic, necroptotic, or necrotic AMs compared with Be-exposed AMs. Exposure of AMs to dexamethasone (DX) for 24 hours in culture induced early apoptosis (EA) and late apoptosis (LA) compared with those cultured with a vehicle (V) control (Figure 1, A and B). TLR ligands, such as LPS, have been shown to induce necroptosis in bone marrow-derived macrophages in the presence of caspase inhibitors (38). Similarly, we found that AMs exposed to LPS with Z-VAD-FMK for 24 hours underwent necrosis (Figure 1, A and B). Death of LZ-exposed cells was impaired in the presence of the RIP1K inhibitor necrostatin-1 (NS) or in the presence of the RIP3K inhibitor GSK'872 (GSK) (Figure 1C), showing that LZ induces necroptosis in AMs. Similar to necroptotic AMs, those exposed to Be particles were necrotic after 24 hours (Figure 1, A and B). Primary necrosis was induced by heat-killing or freeze-thawing AMs (Figure 1B). Analysis of cell-free culture supernatants (SNs) showed that neither DNA nor IL-1 $\alpha$ was released from apoptotic AMs, necroptotic cells released only IL-1 $\alpha$, and cells that died by primary necrosis released only DNA, even in the presence of protease inhibitors (FT+) (Figure 1D). In contrast, Be-exposed AMs released both DNA and IL-1 $\alpha$ upon cell death (Figure 1D). 
A
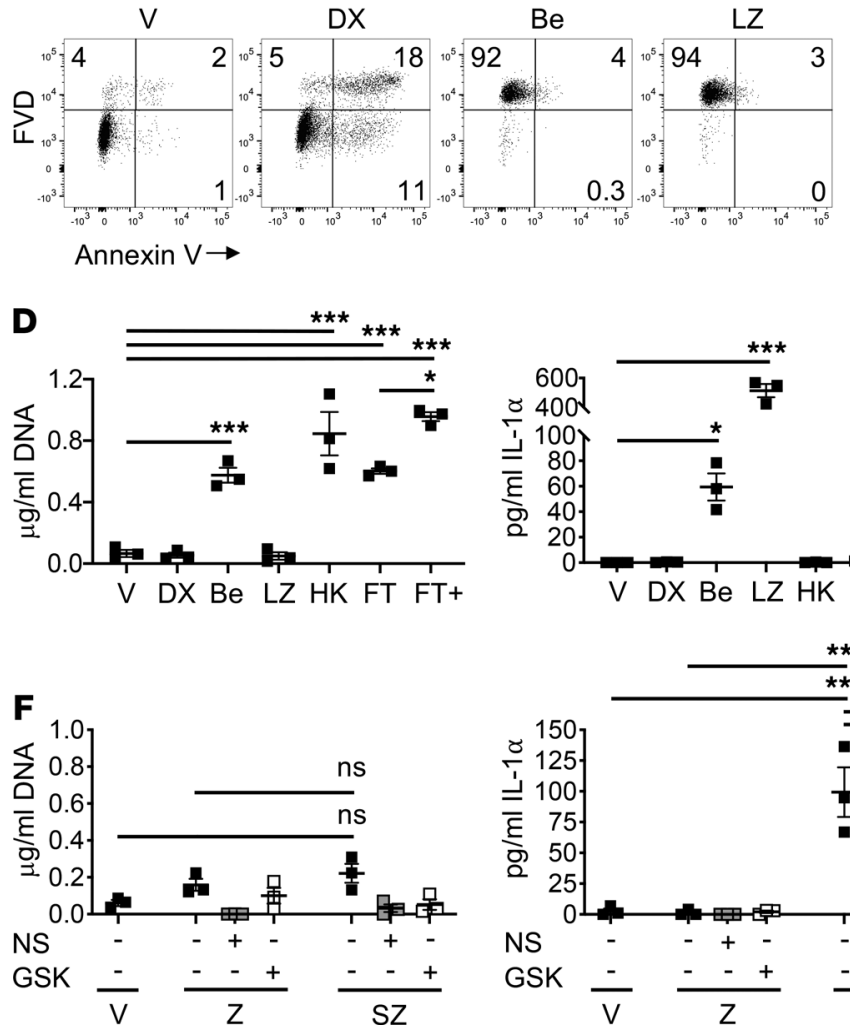
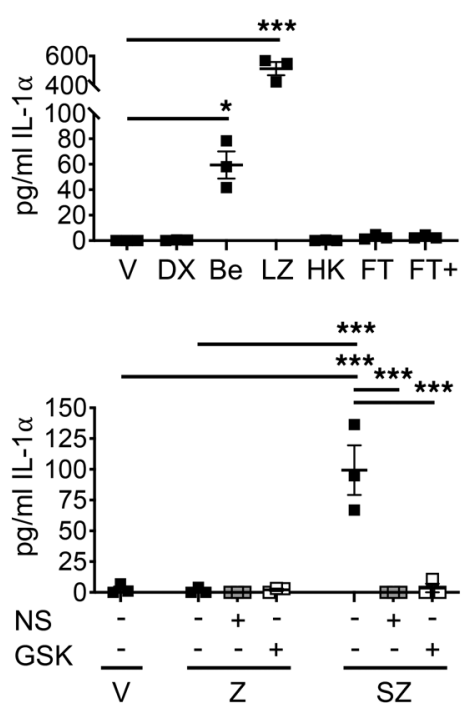

B - EA

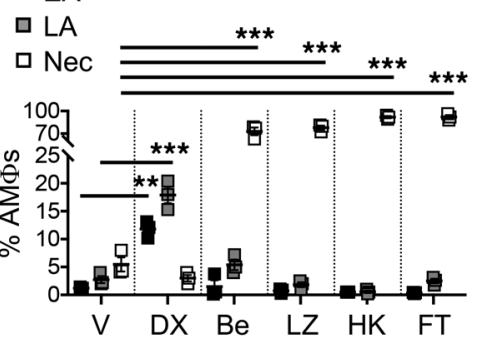

C

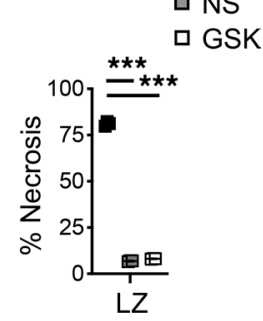

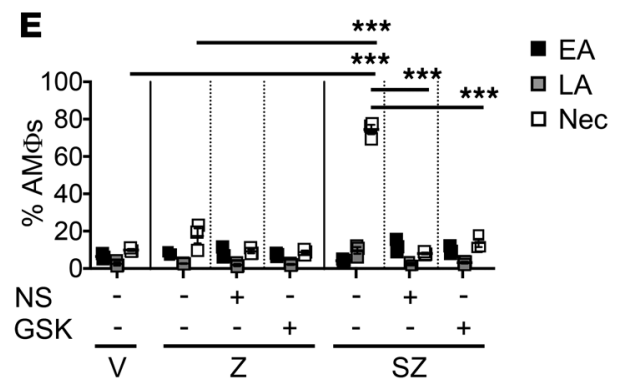

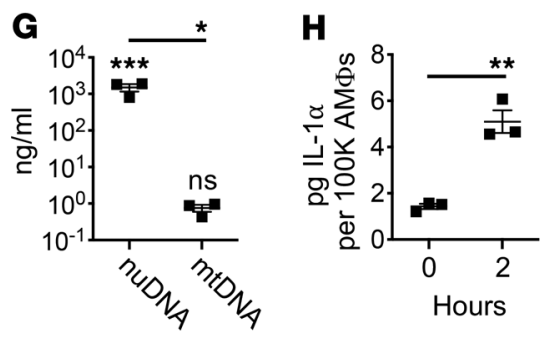

Figure 1. Release of DAMPs from Be-exposed AMs compared with apoptotic, necroptotic, or necrotic AMs. (A-D) AMs from B6 mice were cultured \pm vehicle (V), $100 \mu \mathrm{M}$ dexamethasone (DX), $50 \mu \mathrm{g} / \mathrm{mL}$ Be (Be), or $10 \mu \mathrm{g}$ LPS + $40 \mu \mathrm{M}$ Z-VAD-FMK (LZ) for 24 hours. (A) Gating of early apoptotic (EA), late apoptotic (LA), or necrotic (Nec) cells is shown. Numbers indicate the percentage of AMs in each gate for the sample shown. (B) The percentage of AMs that were EA, LA, or Nec is shown for each treatment group compared with heat-killed (HK) or freeze-thawed (FT) necrotic cells. (C) Percentage of AMs that were Nec following pretreatment with V, $10 \mu \mathrm{M}$ necrostatin-1 (NS), or $0.5 \mu \mathrm{M}$ CSK'872 (CSK) and stimulated with LZ are shown. (D) Concentrations of extracellular DNA and IL-1 $\alpha$ in cell-free supernatants (SNs) are shown for each treatment group. (FT+ indicates protease inhibitors were present). (E and F) B6 AMs were pretreated with V (-), NS, or GSK (as described above) and stimulated \pm V, $40 \mu$ M Z-VAD-FMK (Z) alone or together with $10 \mu M$ CUDC-427 Smac mimetic (SZ) for 24 hours. (E) The percentage of AMs that were EA, LA, or Nec are shown for each group. (F) Concentrations of DNA and IL-1 $\alpha$ in SNs are shown. (C) Mean concentrations of nuclear DNA (nuDNA) and mitochondrial DNA (mtDNA) in bronchoalveolar lavage fluid (BALF) are shown. (H) Intracellular IL-1 $\alpha$ was quantified in lysates of AMs from B6 mice exposed i.t. for either $\mathbf{O}$ or 2 hours to $50 \mu \mathrm{g}$ Be. Data in $\mathbf{A}$ and $\mathbf{C}$ are representative data from 1 of $\mathbf{3}$ independent experiments, symbols on $\mathbf{C}$ are triplicate values, and bars indicate means \pm SD. Data in $\mathbf{B}$ and $\mathbf{D}-\mathbf{H}$ are combined from $\mathbf{3}$ independent experiments and performed in triplicate/experiment (B and $\mathbf{D}-\mathbf{F})$ or $n=5$ mice/experiment $(\mathbf{G}$ and $\mathbf{H})$. Symbols on graphs are mean experimental values, and bars indicate the mean of means \pm SEM. One-way ANOVA (B-G) or an unpaired 2-tailed $t$ test $(\mathbf{H})$ was used to determine statistical differences between groups. Asterisks/ns symbols in $\mathbf{G}$ with no bar were compared with nuDNA or mtDNA quantified from the BALF of PBS-treated control mice. Statistical $P$ values are indicated as ns, not significant; ${ }^{*} P<0.05$; ${ }^{* *} P<0.01 ;{ }^{* *} P<0.001$ for select comparisons.

LPS can directly enhance expression of IL-1 $\alpha$ via TLR4, and therefore DAMP release could differ following TLR-independent necroptosis. Second mitochondria-derived activator of caspase (Smac) is a mitochondrial protein that inhibits cellular inhibitors of apoptotic proteins if released into the cytosol, leading to activation of apoptotic caspases, activation of RIP1K, and initiation of apoptosis (39). When caspase activity is inhibited, as occurs in certain infections or tumor cells, Smac and Smac mimetics promote necroptosis (40-42). We tested whether the Smac mimetic CUDC-427 induced necroptosis of AMs in the presence of the pancaspase inhibitor Z-VAD-FMK (SZ). SZ induced necroptosis that was inhibited by NS or GSK (Figure 1E). Similar to LZ-induced necroptosis, SZ-induced necroptosis did not induce the release of DNA (Figure 1F, left) but did promote IL-1 $\alpha$ release that was RIP1K and RIP3K dependent (Figure 1F, right).

To define the nature of the DNA released by Be-exposed AMs, we used quantitative PCR to determine the copy number and quantity of mitochondrial (mt) or nuclear (nu) DNA (43). This analysis confirmed that the DNA released was nuDNA (Figure 1G). Together, these data suggest that exposure to Be enhances release of both IL-1 $\alpha$ and nuDNA. This profile is similar to that released from AMs in 
response to other crystalline particles (1) and differs from that released by AMs that have undergone apoptosis, necroptosis, or primary necrosis.

Pulmonary exposure to Be particles boosts intracellular stores of $I L-1 \alpha$ in resident AMs. The lack of detectable IL- $1 \alpha$ release by necrotic cells suggested that Be exposure may upregulate intracellular stores of IL- $1 \alpha$ in AMs. Unlike IL-1 $\beta$, IL-1 $\alpha$ is constitutively expressed as a biologically active precursor in many cells and requires enzymatic processing (by caspase-1, for example) to be secreted from living cells $(44,45)$. Many particles induce activation of the cytosolic Nod-like protein NALP3, which triggers assembly of the inflammasome and activation of caspase 1 . We have shown that IL- $1 \alpha$ is necessary and sufficient for IL-1R1dependent neutrophil recruitment that follows pulmonary exposure to $\mathrm{Be}$, and its release into the airways is independent of NALP3 and caspase-1 (12). In addition, IL-1 $\alpha$ levels rise after a drop in AM numbers in Be-exposed mice and is not accompanied by a rise in IL-1 $\beta$ (2). These observations suggest IL-1 $\alpha$ is released as a DAMP from dying AMs and not actively secreted. In monocytic cells, intracellular stores of IL-1 $\alpha$ are low but can be boosted by a variety of TLR ligands, inflammatory cytokines (including TNF- $\alpha$ ), or particles $(7,44,45)$. To test whether Be exposure could have a similar effect on intracellular IL-1 $\alpha$ protein levels in AMs, we harvested AMs from mice exposed intratracheally (i.t.) to Be for 2 hours, a time that precedes the drop in AM numbers in Be-exposed mice, the detection of extracellular IL-1 $\alpha$ in airway fluids, and the onset of neutrophil recruitment (2). AMs were lysed and IL-1 $\alpha$ in cell lysates was quantified by an ELISA. Intracellular IL-1 $\alpha$ was detected at low levels in the lysates of steady-state AMs as predicted by transcriptome data (46) but was increased in the lysates of AMs from Be-exposed mice (Figure 1H).

Be-induced AM cell death is dependent upon phagocytosis. AMs die after exposure to Be crystals in vitro and in vivo (2). A possible cause could be that Be directly affects cell viability by disrupting the plasma membrane. To rule this out, we pretreated purified murine AMs with cytochalasin D (Cyto D) to inhibit actin-mediated phagocytosis and cultured the cells in the presence or absence of Be for 18 hours. Cyto D treatment rescued AMs from Be-induced cell death and prevented their release of extracellular DNA and IL-1 $\alpha$ in SNs (Figure 2A). Thus, Be must be internalized by AMs to trigger death and release immunogenic DAMPs. Phagocytosis can induce ROS production; however, AM cell death, release of DNA and IL- $1 \alpha$, and initiation of airway inflammation were similar in Be-exposed WT and Nadph $p 47^{-1-}$ (p47-KO) mice (Supplemental Figure 1, A-D; supplemental material available online with this article; https://doi.org/10.1172/jci.insight.134356DS1). Similarly, antioxidant treatment had no effect on Be-induced AM cell death or DAMP release in vitro (Supplemental Figure 1E). Therefore, we have thus far found no evidence of the involvement of ROS or ROS-dependent pathways (such as ferroptosis; ref. 47) in Be-induced AM cell death or DAMP release.

Caspase and cathepsin enzyme activities promote DNA release from Be-exposed AMs but are dispensable for Be-induced cell death in AMs. A role for apoptotic caspases in the release of fragmented DNA from dying cells has previously been established (37). To ascertain whether caspase activity, known to be required for pyroptosis and apoptosis/secondary necrosis, plays a role in Be-induced AM cell death or DAMP release, we pretreated AMs with the pancaspase inhibitor Z-VAD-FMK or vehicle control and cultured cells for 18 hours in media with or without Be. Be-induced AM cell death occurred independent of caspase activity (Figure 2B, left). However, caspase activity promoted release of DNA from Be-exposed AMs (Figure 2B, middle). In contrast, release of IL- $1 \alpha$ from Be-stimulated AMs was caspase independent (Figure 2B, right).

$B e$-induced $A M$ cell death and DAMP release did not require the enzymatic activity of RIP1K or RIP3K. Inhibition of necroptosis with either a RIP1K inhibitor (NS) or RIP3K inhibitor (GSK) had no significant impact on Be-induced cell death or DAMP release but prevented LZ-induced necroptosis and associated release of IL-1 $\alpha$ (Figure 2C). To confirm that apoptosis and necroptosis did not have redundant roles in Be-induced AM cell death or DAMP release, we inhibited both pathways in the same cells using combined treatment with Z-VAD-FMK and GSK. This treatment had no impact on Be-induced AM cell death and impaired DNA release, similar to the effects of caspase inhibition alone (Supplemental Figure 2A). Thus, Be-induced caspase activity promotes release of DNA, and necroptotic pathways did not account for Be-induced death or DAMP release in the presence or absence of caspase activity.

Lysosomal destabilization is required for cell death in Be-exposed AMs. Crystalline particles induce leakage of cathepsins B and L into the cytosol, which, in addition to caspases, can promote apoptosis and fragmentation of nuDNA (48). To test whether cathepsins play a role in the release of DNA from Be-exposed AMs, we pretreated AMs with the cathepsin B/L inhibitor Z-FF-FMK, the caspase inhibitor Z-VAD-FMK, or both inhibitors together. Neither inhibitor impaired cell death or IL-1 $\alpha$ release (Supplemental Figure 2B). 

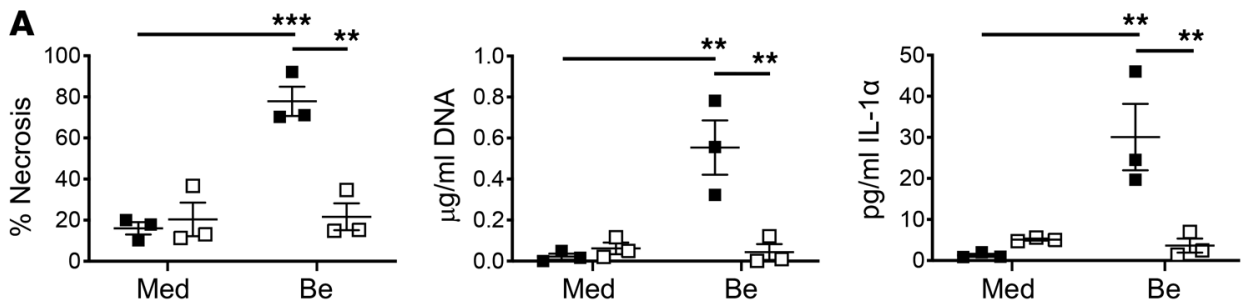

Veh
Cyto D
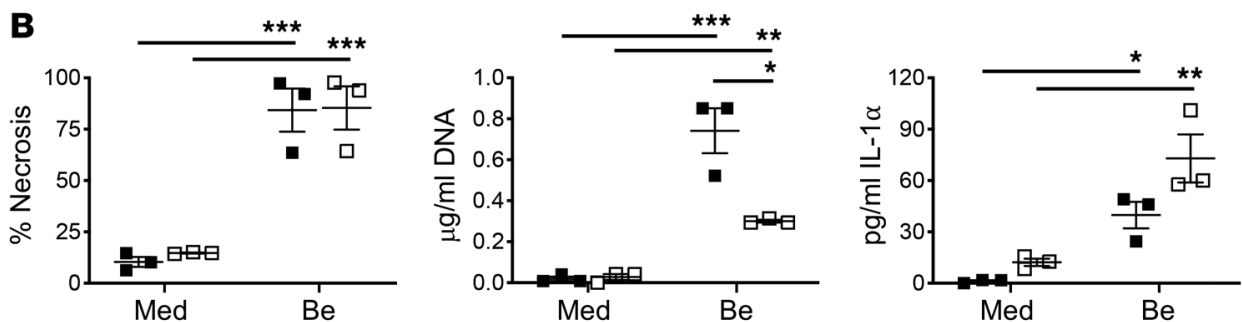

- Veh

口 Z-VAD
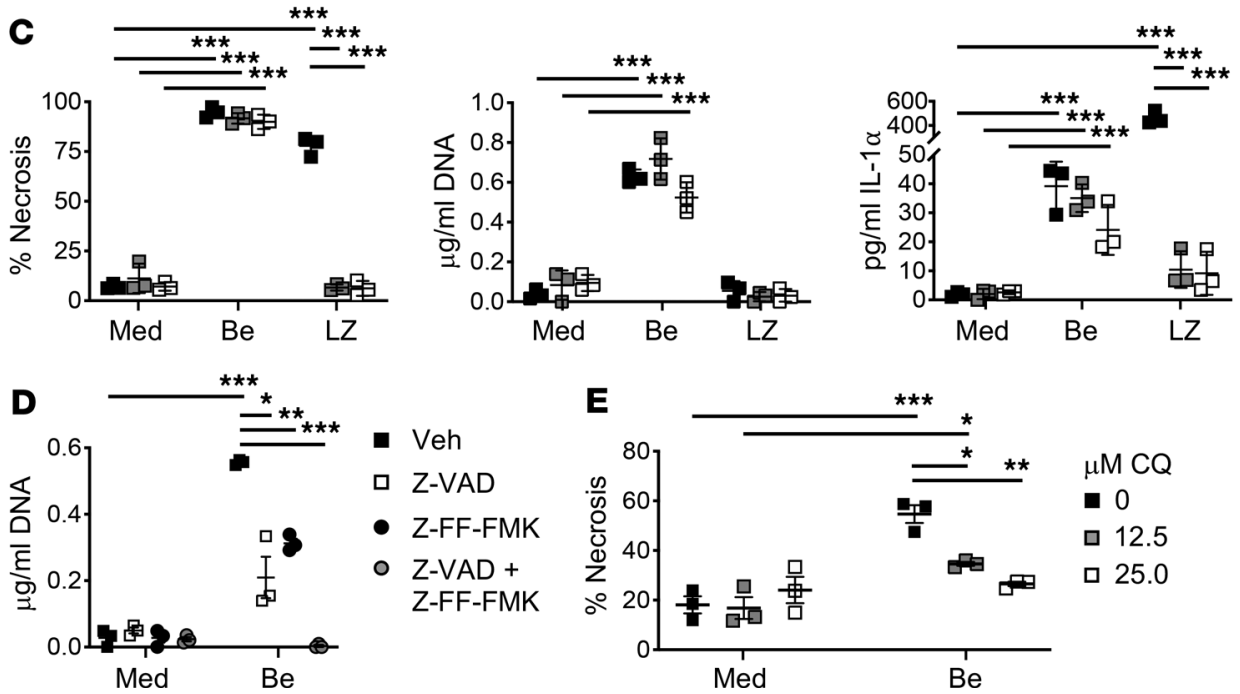

Figure 2. AM cell death and DAMP release are regulated by separate pathways. (A-C) B6 AMs were pretreated with vehicle (Veh) or the indicated inhibitors for 15 minutes and then stimulated $\pm 50 \mu \mathrm{g} / \mathrm{mL}$ Be for 18 hours. AM necrosis and DAMP release were assessed as described in Figure 1. Percentage of AMs that were necrotic (left) and concentrations of DNA (middle) or IL-1 $\alpha$ (right) in culture SNs are shown for each treatment group. (A) AMs were pretreated with Veh or $10 \mu \mathrm{M}$ cytochalasin D (Cyto D). (B) AMs were pretreated with Veh or $20 \mu \mathrm{M}$ Z-VAD-FMK (Z-VAD). (C) AMs were pretreated with Veh $10 \mu \mathrm{M}$ necrostatin-1 (NS), or $0.5 \mu \mathrm{M}$ CSK'872 (CSK). Some samples were stimulated with $10 \mu \mathrm{g} / \mathrm{mL}$ LPS + $40 \mu \mathrm{M}$ Z-VAD-FMK (LZ) as a positive control for necroptosis. (D) AMs were pretreated with vehicle (Veh), $20 \mu \mathrm{M} Z$ Z-VAD-FMK (Z-VAD), $10 \mu \mathrm{M}$ Z-FF-FMK, or Z-VAD + Z-FF-FMK. Concentrations of DNA in SNs are shown for each treatment group. (E) MH-S cells were pretreated with indicated concentrations of chloroquine (CQ) for 1 hour and then exposed to Be for 18 hours. The percentage of MH-S cells that underwent necrosis is shown. Data are combined from 3 independent experiments and performed in duplicate/experiment with separate cohorts of mice or passages of $\mathrm{MH}-\mathrm{S}$ cells as a source of cells in each experiment. Symbols on graphs are mean values from each experiment \pm SEM. A 1-way ANOVA was used to test for statistical differences between groups. Statistical $P$ values for select comparisons are indicated as ${ }^{*} P<0.05 ;{ }^{* *} P<0.01 ;{ }^{* *} P<0.001$

However, Z-FF-FMK and Z-VAD-FMK each reduced DNA release from Be-exposed AMs, and DNA release was completely impaired from the dying cells when Z-FF-FMK and Z-VAD-FMK were used together (Figure 2D). This suggested that Be induced lysosomal leakage. To examine lysosomal leakage using time-lapse microscopy, we used the murine AM-derived MH-S cell line. These cells underwent cell death with similar kinetics as resident AMs and released DNA upon cell death (Supplemental Figure 3A). MH-S cell endosomes and phagosomes were preloaded with FITC-dextran, nuclei were stained with Hoechst, and the cells were imaged by 2-photon microscopy before and after addition of media, $\mathrm{Be}, \mathrm{or}_{\mathrm{SiO}}$ as a positive control (49). We observed a modest increase in FITC fluorescence in the nuclear regions of MH-S cells following addition of Be (Supplemental Figure 3, B and C). This effect was reduced and delayed as compared with $\mathrm{SiO}_{2}$-exposed $\mathrm{MH}$-S cells and was not apparent in the cells exposed to media alone (Supplemental Figure 3B). Furthermore, stabilization of lysosomes by treating MH-S cells with chloroquine (CQ) prevented Be-induced cell death (Figure 2E). 
A

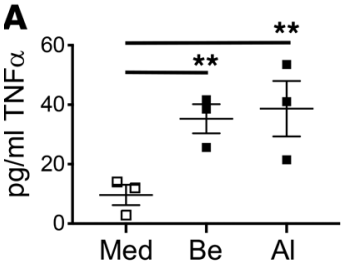

C
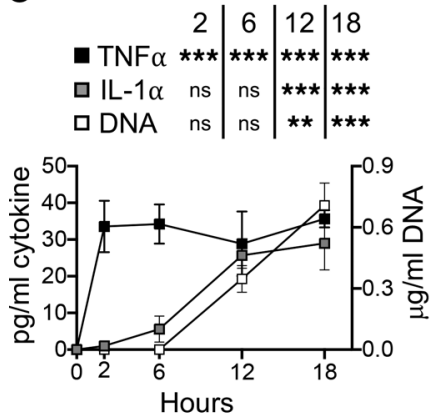

B

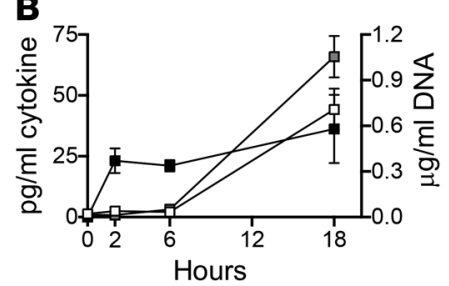

D
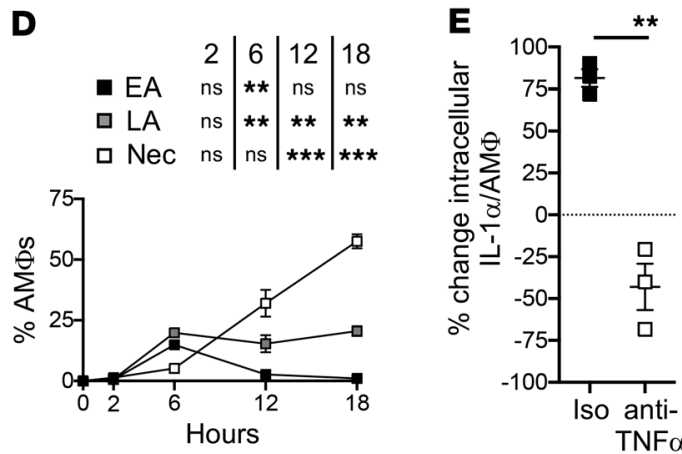

Figure 3. Be particles stimulate rapid secretion of TNF- $\alpha$ from AMs that affects their intracellular levels of IL-1 $\alpha$. (A) AMs from B6 mice were cultured for 18 hours $\pm 50 \mu \mathrm{g} / \mathrm{mL}$ Be (Be) or aluminum hydroxide (Al) for 18 hours. TNF- $\alpha$ concentrations in SNs are shown. (B) TNF- $\alpha$, IL-1 $\alpha$, and DNA in BALF from B6 mice exposed i.t. to Be (normalized to timed PBS-exposed controls) for each time point are shown. Key indicates each parameter and the $P$ value for comparisons between Be- or PBS-exposed mice for the indicated time point. (C and D) B6 AMs were cultured for indicated times in media (Med) \pm Be. Keys indicate each parameter and the $P$ value for comparisons between Be- or Med-exposed AMs for the indicated time point. (C) Concentrations of extracellular TNF- $\alpha$, IL-1 $\alpha$, and DNA in SNs (normalized to timed media controls) are shown for each time point. (D) The percentage of Be-exposed AMs that were early apoptotic (EA), late apoptotic (LA), or necrotic (Nec) (normalized to timed media controls) are shown. (E) B6 mice were treated i.p. with 200 $\mu \mathrm{g}$ isotype control (Iso) or anti-TNF- $\alpha$ antibodies. Cohorts from each group were left untreated or exposed i.t. to $50 \mu \mathrm{g}$ Be/mouse for 2 hours. Intracellular IL-1 $\alpha$ was quantified as described in Figure $1 \mathrm{H}$. Data are expressed as percentage change in IL-1 $\alpha /$ AM due to Be exposure (vs. unexposed controls). Graphs contain data combined from 3 independent experiments. Symbols on dot plot graphs (A and $\mathbf{E}$ ) are experimental mean values, and bars indicate the mean \pm SEM. Symbols on time course graphs (B-D) indicate the mean of 3 experimental means \pm SEM for each time point. Treatments for vitro AM assays (A-D) were performed in duplicate/experiment using separate cohorts of mice as sources of AMs. Symbols in $\mathbf{E}$ are combined from 3 independent experiments ( $n$ $=5$ mice/group in each experiment). One-way ANOVA $(\mathbf{A}-\mathbf{D})$ or an unpaired 2-tailed $t$ test $(\mathbf{E})$ was used to test for differences between groups. $P$ values for selected comparisons are indicated as ${ }^{*} P<0.05 ;{ }^{*} P<0.01 ;{ }^{* *} P<0.001$.

Thus, internalization of Be particles by AMs leads to lysosomal leakage and cell death in the absence of functional apoptosis/necroptosis/ferroptosis programmed cell death pathways.

Be particles induce rapid secretion of TNF- $\alpha$ by AMs that boosts AM intracellular stores of IL-1 $\alpha$. Other crystalline particles, such as aluminum hydroxide (A1), induce lysosome leakage and death of AMs that results in the release of both DNA and IL-1 $\alpha(1,3)$. We observed that both of these particles enhanced release of TNF- $\alpha$ by AMs in vitro (Figure 3A). Furthermore, mice exposed i.t. to Be for 2 hours had elevated levels of TNF- $\alpha$ in the airways, a time point that preceded detection of extracellular IL-1 $\alpha$ or DNA in the BALF (Figure 3B). Similarly, AMs exposed to Be in vitro secreted elevated levels of TNF- $\alpha 2$ hours later, as compared with their release of extracellular IL-1 $\alpha$ and DNA into culture SNs that occurred 12 to 18 hours after Be exposure (Figure 3C). Analysis of cell death in these Be-exposed AMs by flow cytometry (gating shown in Supplemental Figure 4A) indicated that no cell death had occurred 2 hours after exposure, apoptosis was initiated in the cells 6 hours after exposure, and accumulation of necrotic cells occurred 12 to 18 hours after exposure (Figure 3D). The kinetics of necrosis coincided with the accumulation of extracellular IL-1 $\alpha$ and DNA in culture SNs (Figure 3, C and D). Thus, TNF- $\alpha$ is secreted before cell death or release of DAMPs (IL- $1 \alpha$ and DNA). The kinetics of Be-induced TNF- $\alpha$ secretion coincided with increased quantities of intracellular stores of IL-1 $\alpha$ in Be-exposed AMs (Figure 1H). To determine whether elevated levels of intracellular IL- $1 \alpha$ in the AMs from Be-exposed mice were TNF- $\alpha$-dependent, we quantified intracellular IL-1 $\alpha$ in AMs from mice treated systemically with isotype control antibody or TNF- $\alpha$-blocking antibody and exposed i.t. to nothing (0 hours) or Be for 2 hours. Intracellular levels of IL-1 $\alpha$ in AMs of isotype control-treated, Be-exposed mice were increased compared with isotype control-treated, unexposed controls, and this effect was TNF- $\alpha$ dependent (Figure 3E). Together, these data suggest that Be and perhaps other particles that induce rapid secretion of TNF- $\alpha$ from AMs can boost intracellular stores of IL- $1 \alpha$ in an autocrine manner that are later released from the cells upon death. 
A

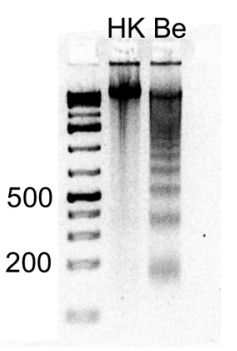

B - Cas8

口 Cas9

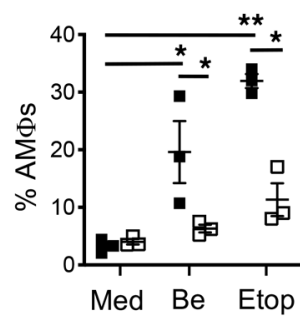

C

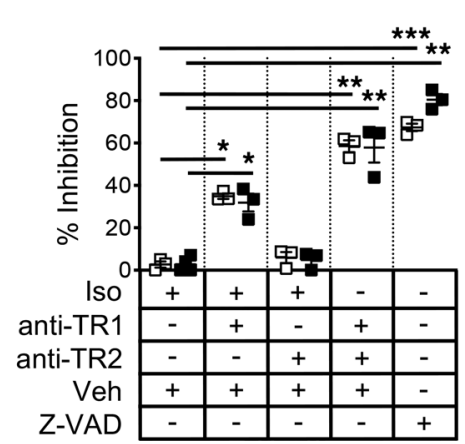

D

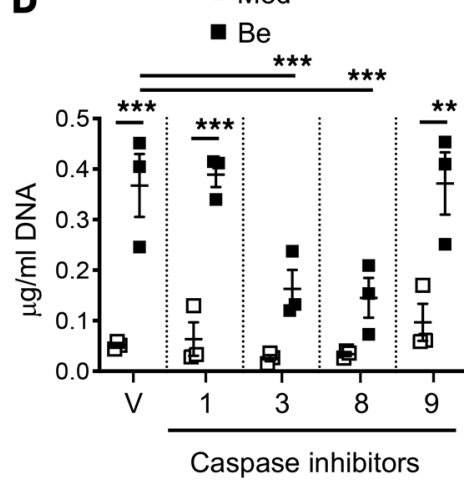

Figure 4. TNF- $\alpha$ promotes release of DNA from Be-exposed AMs by activating caspase-8. (A) Gel electrophoresis of DNA extracted from HK AMs or AMs exposed to Be for 5 hours is shown. Numbers indicate bp as determined by the DNA ladder. Data are from 1 representative of 3 independent experiments. (B) AMs from B6 mice were cultured for 10 hours in media $\pm 50 \mu \mathrm{g} / \mathrm{mL}$ Be or $100 \mu \mathrm{M}$ etoposide (Etop). Ten hours was chosen because we could detect caspase- 8 activation with both stimuli at this time. The percentage of AMs positive for caspase- 8 or caspase- 9 activity is shown. (C) B6 AMs were incubated with $10 \mu \mathrm{g} / \mathrm{mL}$ isotype control or blocking antibodies for TNFR1 (anti-TR1) or TNFR2 (anti-TR2) in media with Vehicle (Veh) or $20 \mu$ M Z-VAD-FMK (Z-VAD) for 30 minutes. The cells were then stimulated $\pm 50 \mu \mathrm{g} / \mathrm{mL}$ Be for 10 hours. Data are expressed as percentage inhibition of Be-induced caspase activity. (D) B6 AMs were pretreated with vehicle $(V), 10 \mu \mathrm{M}$ caspase-1, $10 \mu \mathrm{M}$ caspase- $3,20 \mu \mathrm{M}$ caspase- 8 , or $10 \mu \mathrm{M}$ caspase- 9 inhibitors followed by stimulation \pm 50 $\mu \mathrm{g} / \mathrm{mL}$ Be for 18 hours. Concentrations of DNA in SNs are shown for each treatment group. Graphs contain data combined from 3 independent experiments. Symbols on dot plot graphs are experimental mean values; bars indicate the mean \pm SEM. Treatments for vitro AM assays (B-D) were performed in duplicate/experiment using separate cohorts of mice as sources of AMs. One-way ANOVAs (B-D) were used to test for differences between groups. $P$ values for selected comparisons are indicated as ${ }^{*} P<0.05 ;{ }^{*} P<0.01 ;{ }^{* *} P<0.001$.

TNF- $\alpha$ amplifies release of fragmented DNA from Be-exposed AMs by promoting activation of caspase- $8 . \mathrm{BeSO}_{4}$ has previously been reported to initiate apoptosis in macrophages $(50,51)$, and our data show evidence that uptake of Be by AMs initiates an apoptotic program after secretion of TNF- $\alpha$ (Figure 3, C and D). Our data also show that caspase activity is paradoxically not required for cell death but does promote the release of DNA from the cells upon their loss of membrane integrity. Initiation of the apoptotic program in Be-exposed AMs may fragment the chromatin into nucleosomes that are smaller and thus more easily released from the cells upon their demise. DNA isolated from AMs exposed to Be particles for 5 hours had a distinct 200-bp DNA ladder profile consistent with nucleosomal fragments, in contrast with unfragmented DNA isolated from HK AMs (Figure 4A). Be-exposed AMs had increased caspase- 8 activity but not caspase- 9 activity (Supplemental Figure 4B and Figure 4B), implicating extrinsic apoptosis. Furthermore, incubation of AMs with blocking antibodies against TNF receptors 1 and 2 (TNFR1 and TNFR2) inhibited caspase-3 and -8 activity in Be-exposed AMs, and this inhibition was similar to that observed with Z-VAD-FMK (Figure 4C).

To confirm that the release of DNA was dependent upon caspase- 8 and the downstream activation of caspase- 3 in Be-exposed AMs, we pretreated the cells with specific inhibitors for caspase- $1,-3,-8$, or -9 and cultured the cells with or without Be particles for 18 hours. As predicted by our Z-VAD-FMK data (Figure 2B), none of the caspase inhibitors affected cell death (Supplemental Figure 6), but inhibition of either caspase- 3 or caspase- 8 activity impaired DNA release from Be-exposed AMs upon loss of membrane integrity (Figure 4D). Together, these data suggest that after 2 hours of Be exposure, TNF- $\alpha$ initiates apoptosis via activation of caspase- 8 and caspase-3, leading to fragmentation of the chromatin into nucleosomes. The data suggest that if the apoptotic program is inhibited, DNA is less efficiently released from the dying cells. Prosurvival and proinflammatory signaling via TNFRs shift toward proapoptotic signaling when NF- $\mathrm{B}$ activity drops $(52,53)$. In accordance with this, TNF- $\alpha$-induced NF- $\kappa B$ activity in a reporter macrophage cell line was reduced if cells were pretreated with Be for 2 hours (Supplemental Figure 5). Together these data suggest that TNF- $\alpha$ enhances release of DNA by promoting fragmentation of the chromatin into nucleosomes before Be-induced loss of plasma membrane integrity.

Treatment of mice with neutralizing anti-TNF- $\alpha$ antibodies impairs release of nucleosomal DNA and IL-1a after pulmonary exposure to $\mathrm{Be}$. AMs may respond differently in the close proximity of tissue culture compared with their natural environment in the alveoli of the lung. To determine if TNF- $\alpha$ affects AM death and/or DAMP release in vivo, we treated mice systemically with isotype control or anti-TNF- $\alpha$ antibodies and then exposed them i.t. to PBS with or without Be for 18 hours. Resident AM necrosis was assessed 
A

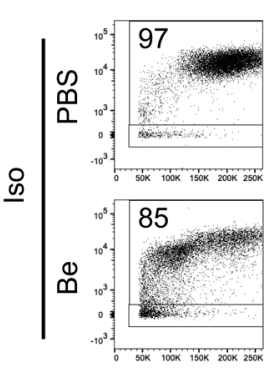

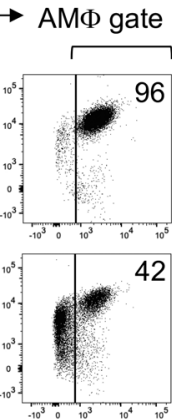
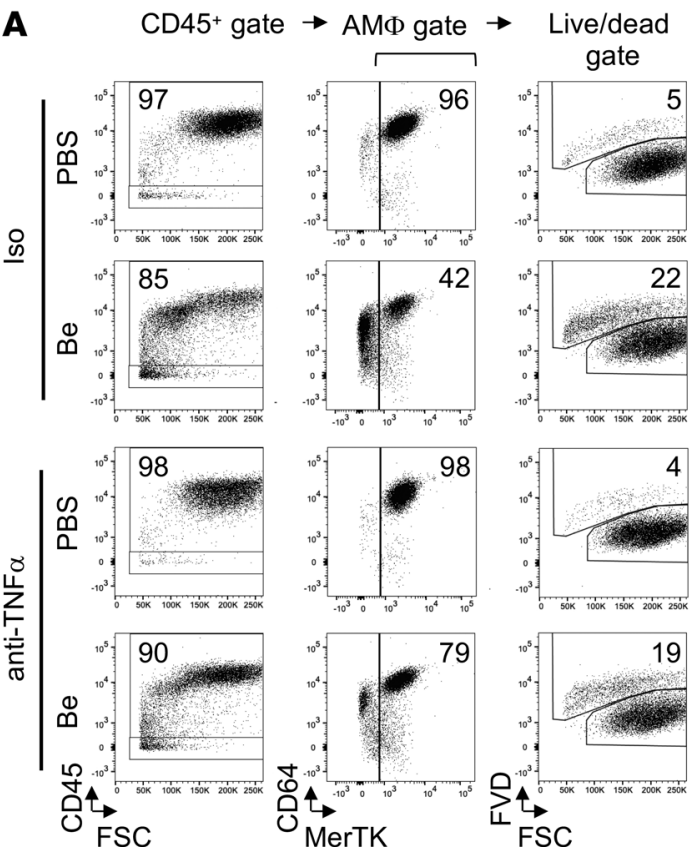

B

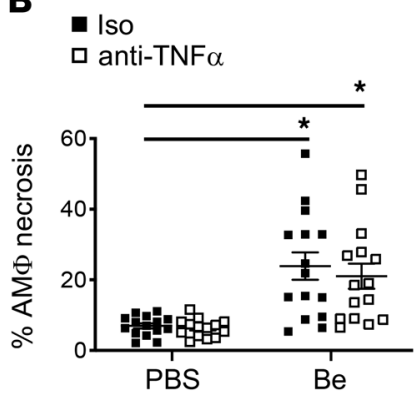

D

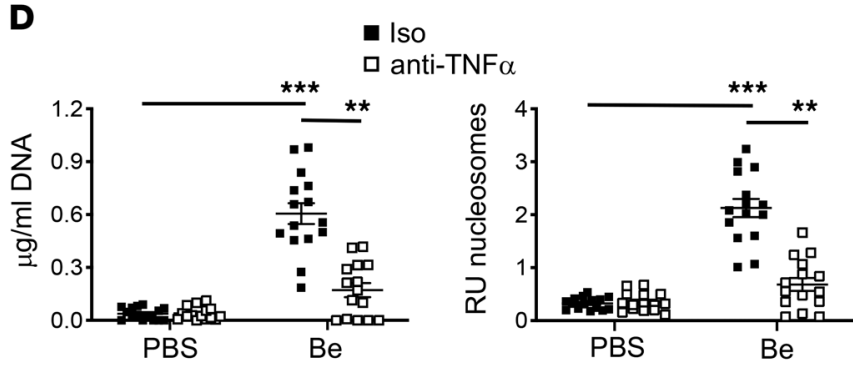

Figure 5. Treatment of mice with anti-TNF- $\alpha$ before pulmonary Be exposure attenuates the release of both DNA and IL-1 $\alpha$ into the airways. B6 mice were injected i.p. with $200 \mu \mathrm{g}$ isotype control or TNF- $\alpha$-blocking antibody. The next day mice were exposed i.t. to PBS $50 \mu \mathrm{g}$ Be. Eighteen hours later mice were sacrificed, BAL cells were harvested, and cells were separated from BALF as described in Methods. (A) Gating strategy for analysis of AM necrosis is shown for representative samples in each group. Numbers on plots indicate the percentage of each gated population (indicated at the top of each column) that fall into the indicated gates. Data are representative samples from 1 of 4 independent experiments. (B) The percentage of AMs that were necrotic in each group is shown. (C) Concentrations of extracellular IL-1 $\alpha$ detected in the BALF are shown for each treatment group. (D) Concentrations of dsDNA detected in the BALF are shown (left). Relative levels of histone-associated (nucleosomal) DNA in the BALF are shown for each treatment group (right). Data in B-D are combined from 4 independent experiments ( $n=15$ mice per treatment group). Symbols on dot plot graphs indicate values from individual mice; bars indicate means \pm SEM. A 1-way ANOVA was used to test for statistical differences between indicated groups. Statistical $P$ values for selected comparisons are indicated as ${ }^{*} P<0.05 ;{ }^{* *} P<0.01 ;{ }^{* *} P<0.001$.

in bronchoalveolar lavage (BAL) cells by flow cytometry (Figure 5A). As predicted by our in vitro experiments, inhibition of TNF- $\alpha$ in vivo had no impact on Be-induced AM cell death (Figure 5, A and B) but did impair the release of both IL-1 $\alpha$ (Figure 5C) and nucleosomal DNA (Figure 5D) into the airway fluids.

Treatment of Be-exposed mice with neutralizing anti-TNF- $\alpha$ antibodies impairs $I L-1 \alpha$-dependent recruitment of neutrophils into the airways and mobilization of immunogenic pulmonary $c D C$ s from the lung to the LDLNs. Neutrophil recruitment in Be-exposed mice is driven by IL-1 $\alpha$ in Be-exposed mice with no evidence of a role for caspase-1, NALP3, or IL-1 $\beta(2,36)$. Therefore, we predicted that neutrophil entry into the airways of Be-exposed mice would be attenuated by anti-TNF- $\alpha$ treatment. Treatment of mice with anti-TNF- $\alpha$ resulted in a reduction in the percentage and total number of neutrophils in the BAL after pulmonary exposure to Be particles (Figure 6, A and B). DNA released from Be-exposed AMs promotes activation and migration of CDCs from the lung to the LDLNs via TLR9, and IL-1 $\alpha$ contributes to the upregulation of costimulatory molecules on $\mathrm{CDCs}$ (2). Disruption of both pathways by treating $\mathrm{Tlr} 9^{-1}$ (TLR9-KO) mice with neutralizing antibodies against IL-1 $\alpha$ or by genetic deficiency of the MyD88 adapter protein ablates these effects $(2,12)$. Therefore, we predicted that treating mice with anti-TNF- $\alpha$, which inhibits both IL-1 $\alpha$ and DNA release, would have similar effects and impair the accumulation of activated $\left(\mathrm{CD} 80^{\mathrm{hi}}\right)$ pulmonary $\mathrm{CDCs}$ in the LDLNs after pulmonary exposure to Be. We analyzed cDCs in the LDLNs of mice that had been treated with an isotype control or anti-TNF- $\alpha$ antibody and exposed them i.t. to PBS or Be particles. The percentage of migratory MHCII ${ }^{\text {hi }} \mathrm{cDCs}$ was increased following Be exposure in isotype-treated but not anti-TNF- $\alpha$-treated mice (Figure 6C, top). CD80 was increased on the surface of $\mathrm{cDCs}$ from Be-exposed isotype control-treated mice, and this was impaired in Be-exposed mice treated with anti-TNF- $\alpha$ (Figure 6C, bottom). The accumulation of total cDCs and immunogenic $\mathrm{CD} 80^{\mathrm{hi}} \mathrm{cDCs}$ in the LDLNs was impaired by the anti-TNF- $\alpha$ antibody treatment in Be-exposed mice (Figure 6D). Anti-TNF- $\alpha$ treatment impaired the recruitment of both pulmonary cDC subsets in Be-exposed mice (Supplemental Figure 7). 
A

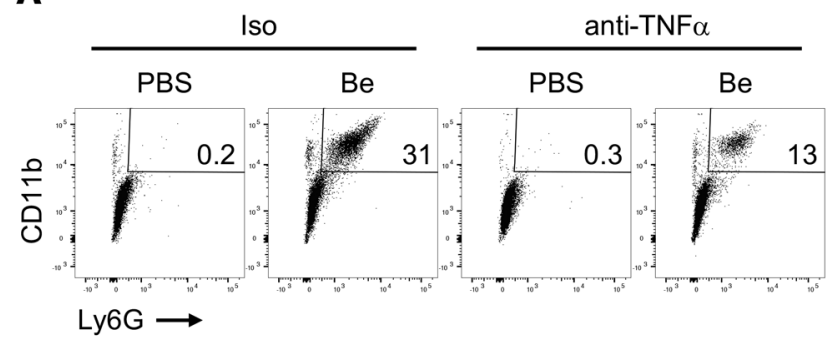

C
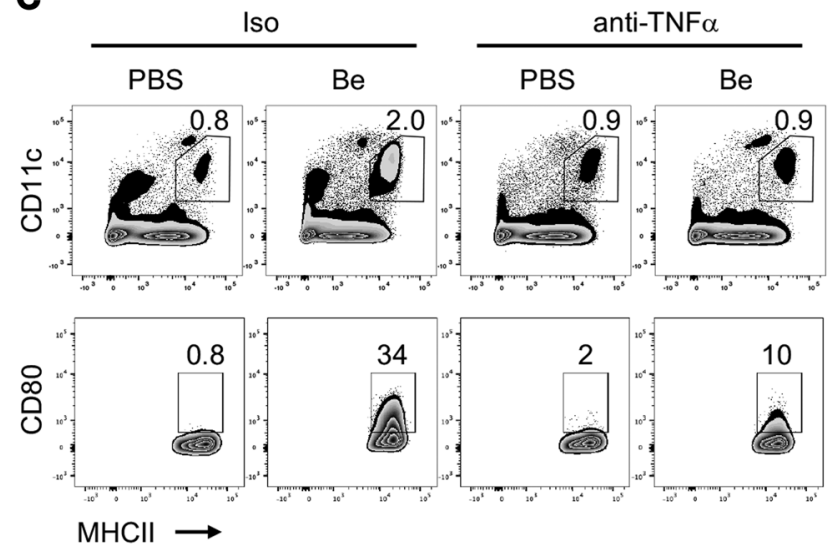

B

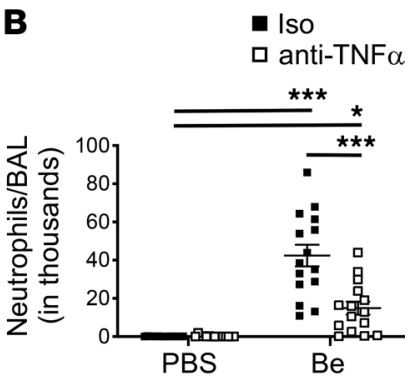

D

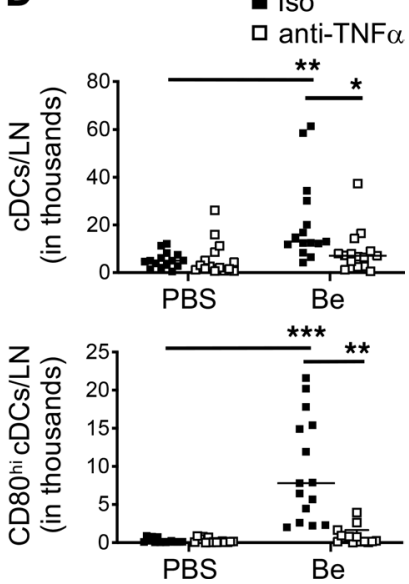

Figure 6. TNF- $\alpha$ is required for neutrophil recruitment to the airways and for mobilization of activated pulmonary cDCs to the LDLNs of Be-exposed mice. B6 mice were injected i.p. with $200 \mu \mathrm{g}$ isotype control or TNF- $\alpha$ blocking antibody. The next day mice were exposed i.t. to PBS $\pm 50 \mu \mathrm{g}$ Be. Eighteen hours later mice were sacrificed. (A and B) BAL cells were analyzed for neutrophil infiltration, and (C and D) LDLNs were analyzed for CD80 hi $C D C s$. (A) Live CD45+ BAL cells were analyzed by flow cytometry for expression of Ly6C and CD11b; gating of Ly6C ${ }^{\text {hic }}$ CD11 b ${ }^{\text {hi }}$ neutrophils is shown on representative samples from each treatment group. Numbers on plots indicate the percentage of (live) CD45+ BAL cells that fall in the neutrophil gate for the samples shown. (B) Total number of neutrophils/BAL is shown. (C) Percentage of live LDLN CD45+ cells that are migratory CDCs (MHCII ${ }^{\text {hiCD11 }} \mathrm{c}^{+}$) (upper row) and percentage of migratory cDCs that are CD80 hi (lower row) are shown for representative samples in each treatment group. Numbers are the percentage of the gated population that fall into indicated gates for the samples shown. (D) Total migratory cDCs (top) and CD80 ${ }^{\text {hi }} \mathrm{CDCs}$ (bottom) are shown. Data in $\mathbf{A}$ and $\mathbf{C}$ are from 1 representative of 4 independent experiments. Data in $\mathbf{B}$ and $\mathbf{D}$ are combined from 4 independent experiments ( $n=15$ mice/group). Symbols on graphs indicate values from individual mice. Bars indicate means \pm SEM in $\mathbf{B}$ and medians in $\mathbf{D}$. A 1-way ANOVA was used to test for statistical differences between groups in $\mathbf{B}$. A Kruskal-Wallis test was used to test for statistical differences in $\mathbf{D}$. Statistical $P$ values for select comparisons are indicated as ${ }^{*} P<0.05 ;{ }^{* *} P<0.01 ;{ }^{* *} P<0.001$.

Be-induced release of DAMPs, and their effects on neutrophils and cDCs, are impaired in TNF- $\alpha$-deficient mice. To confirm the role of TNF- $\alpha$ in AM DAMP release, we exposed B6 WT and Tnf ${ }^{-}$(TNF- $\left.\alpha-\mathrm{KO}\right)$ mice i.t. to Be and assessed DAMP release and neutrophil accumulation in the airways 24 and 48 hours later compared with unexposed mice. The levels of extracellular DNA and IL-1 $\alpha$ were impaired in the airways of Be-exposed TNF- $\alpha-\mathrm{KO}$ mice compared with similarly exposed WT mice (Figure 7A). Accordingly, Be-induced recruitment of neutrophils to the airways of TNF- $\alpha-\mathrm{KO}$ mice was impaired compared with Be-exposed WT mice (Figure 7B). Analysis of the LDLNs revealed that TNF- $\alpha$ was required for the mobilization of activated pulmonary cDCs from the lung to the LDLNs (Figure 7, C and D)

cDCs and MyD88-dependent signaling are required for expansion of $\mathrm{Be}^{2+} /$ self-peptide-specific $C D 4^{+} T_{\text {eff }}$ cells and for development of pulmonary inflammation in a mouse model of CBD. Engagement of TLR9 and IL-1R1 by DNA and IL-1 $\alpha$, respectively, induces MyD88-dependent mobilization of CD80 ${ }^{\mathrm{hi}} \mathrm{cDCs}$ to the draining lymph nodes in Be-exposed B6 mice, an event associated with enhanced CD4 ${ }^{+} \mathrm{T}$ cell responses to bystander foreign antigens $(2,12)$. To confirm that $\mathrm{cDCs}$ and their mobilization via DAMP/MyD88 signaling pathways were required for the expansion of $\mathrm{Be}^{2+} /$ self-peptide-reactive $\mathrm{CD} 4^{+} \mathrm{T}$ cells, we used a mouse model of CBD. Mice that express both the $\alpha$ and $\beta$ chains of the human HLA-DP2 molecule under control of the mouse MHCII promoter (DP2 mice) develop $\mathrm{Be}^{2+} /$ self-peptide-reactive $\mathrm{CD} 4{ }^{+} \mathrm{T}$ cell responses and pulmonary inflammation characteristic of CBD (26). First, we tested whether depletion of cDCs affected expansion of $\mathrm{Be}^{2+} /$ self-peptide-reactive $\mathrm{CD}^{+} \mathrm{T}$ cells. We generated chimeric mice in which all hematopoietic cells (HCs) and stromal cells (SCs) expressed DP2 and in which only cDCs expressed the human diphtheria 
A

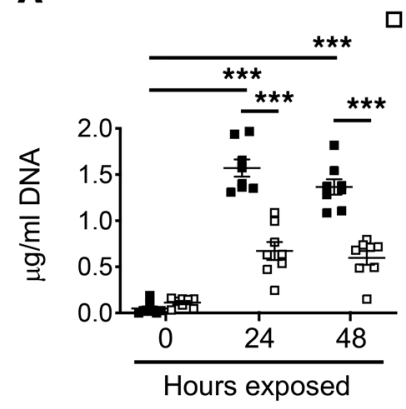

- WT

口 TNFaKO

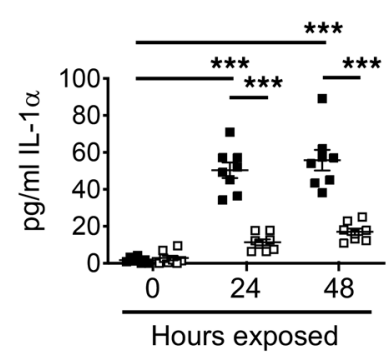

C

- WT

口 TNF $\alpha$ KO
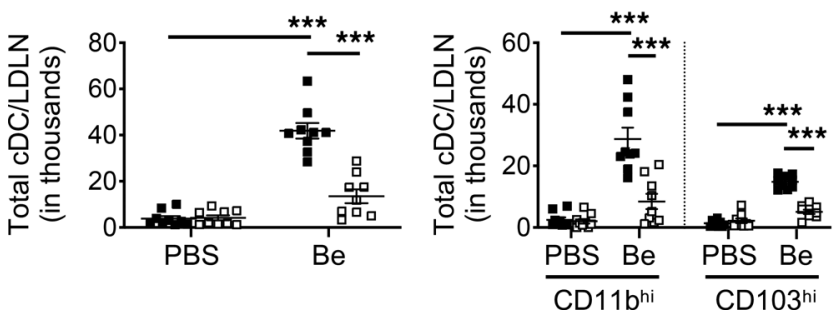

B

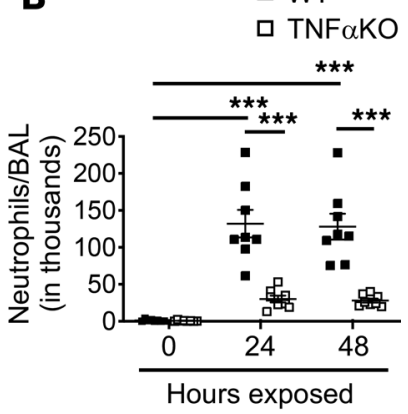

D $\mathrm{WT}$

口 TNF $\alpha$ KO

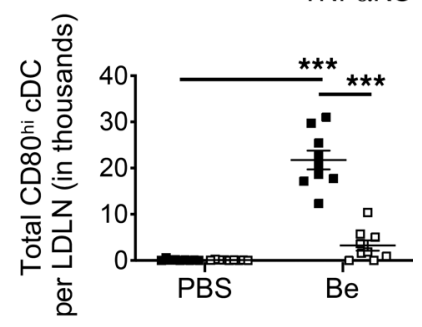

Figure 7. Be-induced DAMP release, pulmonary inflammation, and mobilization of immunogenic cDCs from the lung to the LDLNs are impaired in TNF- $\alpha-K 0$ mice. (A and B) B6 WT and TNF- $\alpha-K O$ mice were exposed i.t. to nothing (0 hours exposed) or to $50 \mu g$ Be i.t. At the indicated times after exposure, BAL cells were analyzed as described in Figure 6A. (A) Concentrations of DNA and IL-1 $\alpha$ detected in the BALF are shown. (B) Total number of neutrophils/BAL are shown for each time point. (C and D) B6 WT and TNF- $\alpha-K O$ mice were exposed i.t. to PBS $\pm 50 \mu g$ Be i.t. for 48 hours. LDLNs were analyzed as described in Figure 6C. (C) Total migratory cDCs per lymph node (LN) and total number of CD11 ${ }^{\text {hi }}$ and CD103 ${ }^{\text {hi }}$ DCs/LN are shown. (D) Total CD80 $0^{\text {hi }} c D C s /$ LN are shown for each treatment group. Data are combined from 2 independent experiments ( $n=8$ mice group in $\mathbf{A}$ and $\mathbf{B} ; n=9$ mice/group in $\mathbf{C}$ and $\mathbf{D})$. Symbols on graphs indicate values from individual mice; bars indicate means $\pm \mathrm{SEM}$. A 1-way ANOVA was used to test for statistical differences between indicated groups. Statistical $P$ values for selected comparisons are indicated as ${ }^{*} P<0.05 ;{ }^{* *} P<0.01 ;{ }^{* *} P<0.001$.

toxin receptor (DTR). This was achieved by reconstituting irradiated DP2 mice with bone marrow from mice that express the DTR under control of the Zbtb46 promoter (zDC-DTR mice). In such mice, cDCs can be specifically depleted upon injection of diphtheria toxin (DT) without toxic effects on cardiac cells or depletion of other monocytic cells $(54,55)$. Offspring from an $\mathrm{F}_{1}$ cross of DP2 and zDC-DTR strains $(54$ 55 ) were used as bone marrow donors for lethally irradiated DP2 mice (Supplemental Figure 8A). Successful immune reconstitution and chimerism were validated in these mice by analyzing the blood cells 8 weeks after reconstitution (Supplemental Figure 8, B and C). Cohorts of chimeric mice were injected i.p. with $\mathrm{PBS} \pm \mathrm{DT}$ on day 0 and every 3 days for the duration of the experiment to maintain $\mathrm{cDC}$ depletion. Mice were then exposed i.t. to PBS or Be on day 0 and sacrificed 12 days later. Depletion of cDCs was successful in the chimeric mice injected with DT (Figure 8A), and expansion of $\mathrm{Be}^{2+} /$ self-peptide-reactive $\mathrm{CD} 4^{+} \mathrm{T}$ cells was eliminated in DC-depleted mice (Figure 8B).

MyD88 signaling (downstream of both TLR9 and IL-1R1) is required for mobilization of CD80 DCs from the lung to the LDLNs after Be-induced DAMP release $(2,12)$. Using bone marrow chimeras in which either SCs or HCs lacked the ability to signal through MyD88, we determined that MyD88-dependent receptor signaling in HCs was required for Be-induced mobilization of cDCs from the lung to the LDLNs (Figure 8C).

We then generated MyD88-deficient DP2 mice to confirm the role of $\mathrm{cDC}$ mobilization in the expansion of $\mathrm{Be}^{2+}$ /self-peptide-specific $\mathrm{CD}^{+} \mathrm{T}$ cells (Supplemental Figure 9). Because HC MyD88dependent signaling is required for the mobilization of activated $\mathrm{CDCs}$ in Be-exposed mice, we gener-

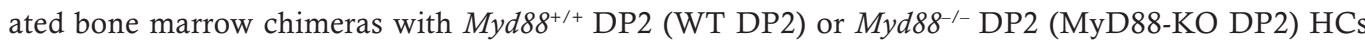
and WT DP2 SCs. Mice were exposed i.t. to either PBS or Be particles on day 0 to promote expansion of $\mathrm{Be}^{2+} /$ self-peptide-specific $\mathrm{CD} 4^{+} \mathrm{T}$ cells. To elicit infiltration of effector $\mathrm{T}$ cells and pulmonary inflammation, all mice were challenged i.t. with $50 \mu \mathrm{g} \mathrm{BeO}$ on days 14 and 16. Mice were sacrificed on day 21 to analyze the presence of $\mathrm{Be}^{2+} /$ self-peptide-reactive $\mathrm{CD} 4^{+} \mathrm{T}$ cells in the lung by ELISPOT and the presence of pulmonary inflammation by histology. Be-exposed mice lacking the ability to signal 
A $\square$ PBS

- $\mathrm{Be}$

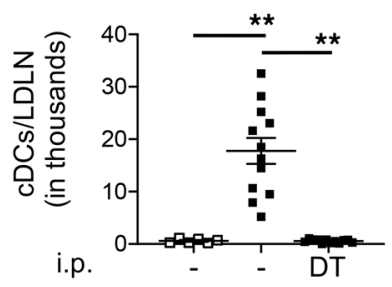

B $\square$ PBS

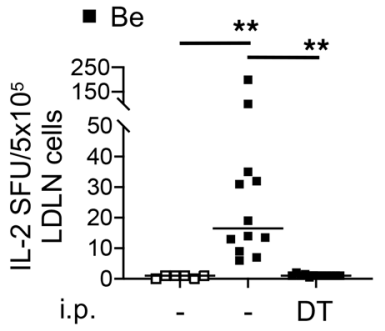

\section{C $\square$ PBS}

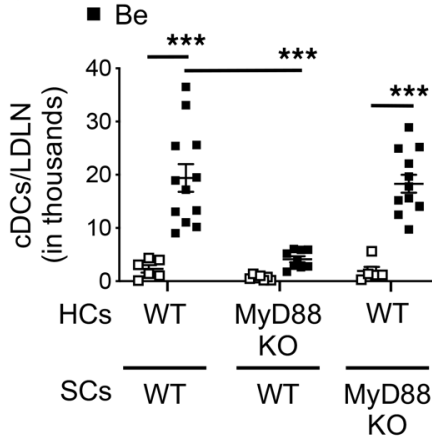

D

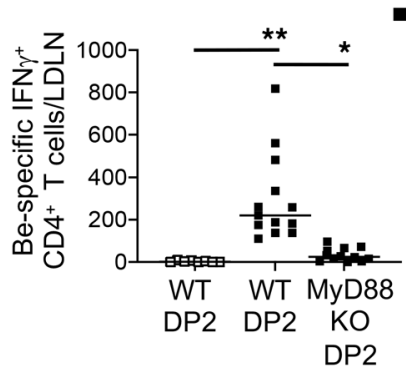

$\mathbf{F}$

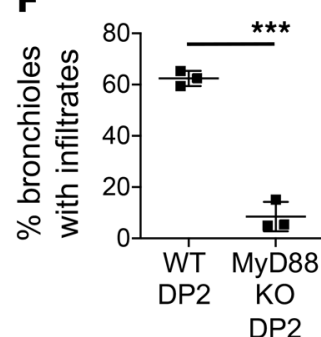

$\square$ PBS

- $\mathrm{Be}$

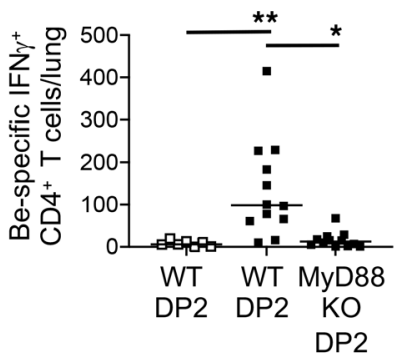

$\mathbf{E}$

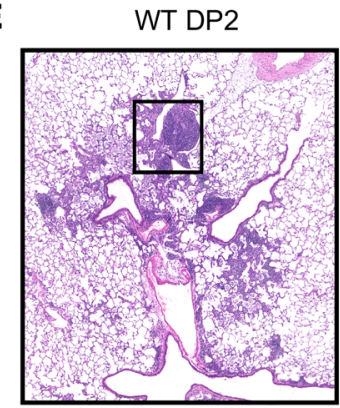

MyD88KO DP2

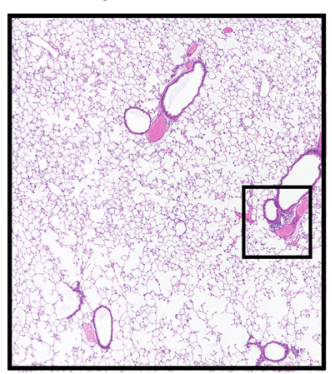

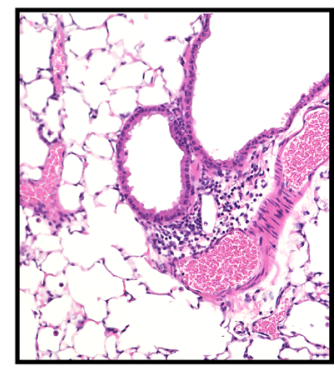

Figure 8. CDCs and MyD88-dependent signaling are required for expansion of $\mathrm{Be}^{2+} /$ self-peptide-specific $\mathrm{CD4} 4^{+} \mathrm{T}$ cell responses in a mouse model of CBD. (A and B) cDCs in zDC-DTR DP2 bone marrow chimeras were depleted in some mice by i.p. injection of diphtheria toxin (DT) every 3 days. Control and cDC-depleted mice were exposed i.t. to PBS or $50 \mu \mathrm{g}$ Be and LDLNs were analyzed 12 days later. (A) Total cDCs/LDLN and (B) IL-2 spot-forming units (SFU) per $5 \times 10^{5}$ LDLN cells are shown. (C) Lethally irradiated B6 (WT or MyD88-KO) mice were reconstituted with B6 (WT or MyD88-KO) bone marrow. Chimeric mice were exposed i.t. to PBS or $50 \mu \mathrm{g}$ Be and accumulation of CDCs in the LDLNs determined 48 hours later as described in Figure 6 . Chimeras with indicated WT or MyD88-KO hematopoietic cells (HCs) and stromal cell (SCs) are indicated. (D and E) FVB/N (WT) DP2 recipients were lethally irradiated and reconstituted with bone marrow from FVB/N DP2 Myd88+/+ (WT DP2) or Myd88/-- (MyD88-KO DP2) littermates. Chimeric mice were exposed i.t. to PBS or $50 \mu \mathrm{g}$ Be on day 0, challenged on day 14 with $\mathrm{BeO}$, and sacrificed on day 21. (D) Total numbers of Be ${ }^{2+} /$ self-peptide-specific IFN- $\gamma^{+}$CD4+ cells detected by ELISPOT in the LDLNs and lungs are shown. (E) H\&E-stained lung sections (original magnification, $\times 4$, top) and indicated areas of lymphocyte infiltration (original magnification, $\times 40$, bottom) are shown. (F) The percentage of bronchioles in each lung with 1 or more peribronchovascular infiltrates is shown. (C) The diameter/infiltrate calculated as described in Supplemental Methods is shown. Data in $\mathbf{A}-\mathbf{D}$ are combined from 2 independent experiments ( $n=12$ mice per group), symbols on graphs are values from individual mice, and bars indicate means \pm SEM (CDC graphs) or medians (ELISPOT data). Data in $\mathbf{E}$ and $\mathbf{G}$ are representative data of 2 independent experiments. Data in $\mathbf{F}$ are combined from 2 independent experiments ( $n=3$ mice/group); bars indicate mean \pm SEM. One-way ANOVA or Kruskal-Wallis tests were used to test for differences between multiple groups of data with a normal (DC data) or skewed (ELISPOT data) distribution, respectively. An unpaired 2-tailed $t$ test was used in $\mathbf{F}$ and $\mathbf{G}$. Statistical $P$ values for selected comparisons are indicated as ${ }^{*} P<0.05 ;{ }^{* *} P<0.01$; ${ }^{* * *} P<0.001$.

through MyD88 in HCs did not mount a $\mathrm{CD}^{+} \mathrm{T}$ cell response to $\mathrm{Be}^{2+} /$ self-peptides (Figure 8D), and $\mathrm{T}$ cell-mediated lung inflammation was reduced in these mice compared with Be-exposed mice with MyD88-sufficient HCs (Figure 8, E-G). Together, these data confirm that the MyD88-dependent effects on cDCs are mediated by receptors on cells in the HC compartment and that MyD88-dependent effects on $\mathrm{CDCs}$ are required to break peripheral tolerance to $\mathrm{Be}^{2+} /$ self-peptides in a mouse model of CBD.

$T N F-\alpha$ blockade prevents expansion of $B e^{2+} /$ self-peptide-reactive $C D 4^{+} T_{\text {eff }}$ cells and prevents $T$ cell-dependent pulmonary inflammation in a mouse model of $C B D$. The requirement for TNF- $\alpha$ to promote migration of immunogenic $\left(C D 80^{\mathrm{hi}}\right) \mathrm{DCs}$ from the lung to the LDLNs in Be-exposed mice (Figures 6 and 7) suggested that neutralization of TNF- $\alpha$ would prevent $\mathrm{Be}^{2+} /$ self-peptide-reactive $\mathrm{T}$ cell responses in a mouse model of CBD. To test this, DP2 
A

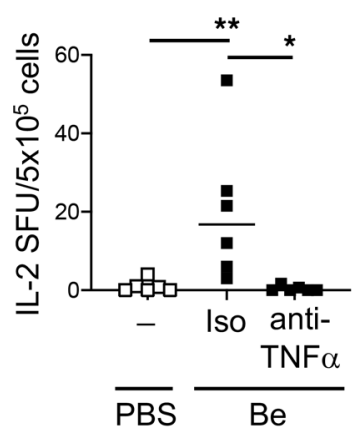

B
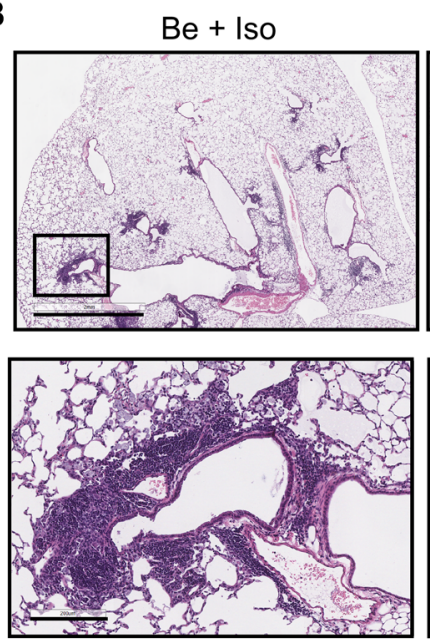

$\mathrm{Be}+$ anti-TNF $\alpha$
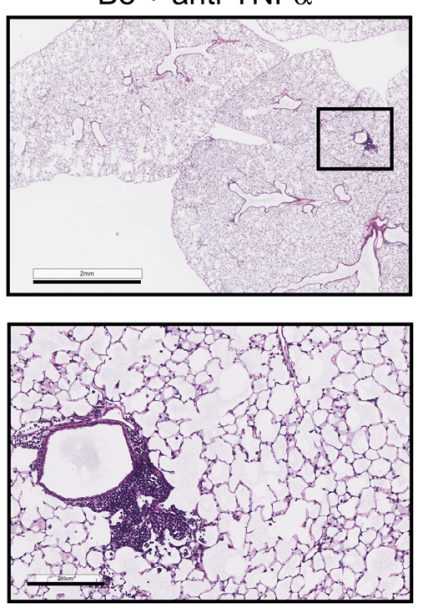

C

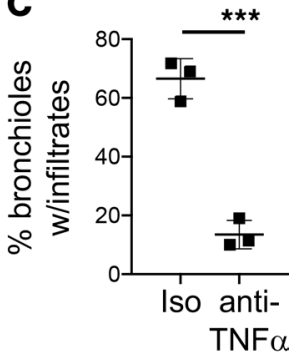

D

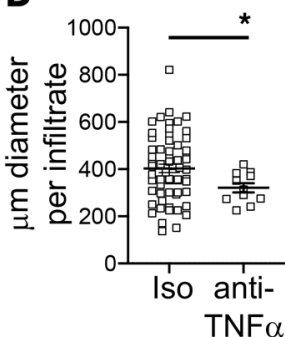

Figure 9. TNF- $\alpha$ is required to break peripheral tolerance and promote lung disease in a mouse model of CBD. FVB/N DP2 mice were injected i.p. with $50 \mu \mathrm{g}$ isotype control antibody, anti-TNF- $\alpha$, or nothing on days $-1,2,5$, and 8 . On day 0 mice were exposed i.t. to PBS or $50 \mu g$ Be, followed by i.t. challenge with $\mathrm{BeO}$ in all groups 2 weeks later, and mice were sacrificed on day 21. (A) The presence of Be ${ }^{2+} /$ self-peptide-reactive CD4 ${ }^{+} \mathrm{T}$ cells in the lungs of mice as detected by IL-2 ELISPOT is shown. The data are expressed as the number of IL-2 spot-forming units (SFU) $/ 5 \times 10^{5}$ lung cells per well. (B) Representative lung scans (top, scale bar: $2 \mathrm{~mm}$ ) and magnified images of the indicated regions, shown in the bottom (scale bars: $200 \mu \mathrm{m}$ ), of H\&E-stained lung sections showing peribronchovascular lymphocyte infiltration (boxes, top) in Be-exposed mice treated with isotype control or anti-TNF- $\alpha$ antibodies. (C) The percentage of bronchioles/section with at least 1 peribronchovascular infiltrate is shown. (D) The diameter of each infiltrate calculated as described in Supplemental Methods is shown. Data in A are combined from 2 independent experiments ( $n=6$ mice per group); bars indicate medians. Data in B are representative of $\mathbf{2}$ independent experiments, and data in $\mathbf{C}$ are combined from 2 independent experiments ( $n=3$ mice per group); bars indicate means \pm SEM. A Kruskal-Wallis test was used to test for statistical differences between groups in $\mathbf{A}$, and an unpaired 2-tailed $t$ test was used to test for differences between groups in $\mathbf{C}$ and $\mathbf{D}$. Statistical $P$ values for selected comparisons are indicated as ${ }^{*} P<0.05 ;{ }^{* *} P<0.01 ;{ }^{* *} P<0.001$.

mice were treated i.p. with $50 \mu \mathrm{g}$ of either an isotype control antibody or anti-TNF- $\alpha$ on days -1 and $1,2,5$, and 8. Mice were exposed i.t. to either PBS or Be particles on day 0 to promote expansion of $\mathrm{Be}^{2+} /$ self-peptide-specific $\mathrm{CD}^{+} \mathrm{T}$ cells. Mice were then challenged by $\mathrm{BeO}$ on days 14 and 16 as described above. Expansion of $\mathrm{CD} 4^{+}$ $\mathrm{T}$ cells reactive to $\mathrm{Be}^{2+} /$ self-peptide was evident in the LDLNs and lungs of Be-exposed DP2 mice treated with the isotype control antibody but not in Be-exposed DP2 mice that received the TNF- $\alpha$-blocking antibody (Figure 9A). Analysis of the lungs by H\&E staining showed that the number and size of peribronchovascular lymphocyte infiltrates were reduced in Be-exposed DP2 mice treated with anti-TNF- $\alpha$ compared with Be-exposed, isotype-treated DP2 mice (Figure 9, B-D). These data show that blocking TNF- $\alpha$ during the initial exposure to Be prevents expansion of $\mathrm{Be}^{2+} /$ self-peptide-reactive $\mathrm{CD} 4^{+} \mathrm{T}$ cells upon secondary exposure to beryllium.

Together, the data in this study show that phagocytosis of sterile crystalline particles by resident AMs induces early secretion of TNF- $\alpha$ that regulates the release of the immunogenic DAMPs IL- $1 \alpha$ and nucleosomal DNA. The data indicate a novel early mechanism for the association of pulmonary environmental exposures and TNF- $\alpha$ in CBD, T cell-mediated hypersensitivities, and autoimmune diseases.

\section{Discussion}

Amorphous crystalline particles induce lysosomal leakage, cellular stress, and/or cell toxicity, and the outcome is cell type dependent and can lead to cell death $(1,5,7,48,56-67)$. We found that Be particle entry into and destabilization of phagolysosomes was required for Be-induced cell death of AMs. This lysosome-associated necrosis occurred with delayed kinetics and was apparent between 12 and 24 hours of Be exposure. This observation may in part explain why Be and other metal crystalline particles are toxic to macrophages but are not toxic to lymphocytes or other nonphagocytic cells (68). The delayed kinetics of Be-induced AM death and requirement for phagocytosis suggested that active processes inside the cell may contribute to cell death. ROS have been implicated in $\mathrm{BeSO}_{4}$-induced $\mathrm{AM}$ apoptosis (69) and are required for execution of ferroptosis. $\mathrm{Be}^{2+}$ ions are released inside the low-pH environment of lysosomes (70) and can interact with ferritin (71), suggesting the possibility that ferroptosis could play 
a role in Be-induced cell death. However, we have thus far found no evidence that Be particles induced or required ROS for Be-induced cell death or IL-1 $\alpha$ release from AMs. It has been postulated that necroptosis and/or apoptosis may be involved in the death of AMs exposed to crystalline particles (1); however, our analysis of the role of caspases, cathepsin B/L, RIP1K, and RIP3K revealed that they were not required for Be-induced loss of plasma membrane integrity in AMs but did regulate DAMP release as discussed below. Thus, our data suggest that Be-induced cell death in AMs was independent of pyroptosis, apoptosis/secondary necrosis, necroptosis, and ferroptosis.

Other studies have shown that primary necrosis can result in different types of DAMPs being released from cells as compared with programmed necrosis (37). Our data show the presence of constitutive levels of IL- $1 \alpha$ in steady-state AMs; however, in Be-exposed mice TNF- $\alpha$ amplified intracellular stores of this cytokine before death of the cells. AMs treated with a caspase inhibitor underwent necroptosis in response to the TLR ligand LPS or a Smac mimetic, and both necroptotic stimuli induced release of IL-1 $\alpha$ but did not release significant levels of DNA. It is possible that the small channels formed by MLKL octamers in necroptotic cells are not large enough for certain molecules, such as unfragmented chromatin, to be efficiently released as compared with HK or FT cells. Analysis of the role of caspases and cathepsins indicated their activity promoted release of fragmented nucleosomal DNA from Be-exposed AMs. Another study showed an essential role for caspase activity in promoting DNA release in response to staurosporine, despite the cells undergoing morphological necrosis (37). Similar to our study, caspases were not required for death but promoted the release of DNA in the form of nucleosomes via a mechanism that may involve chloride ion channels. Studies with radioactive $\left[{ }^{7} \mathrm{Be}\right]$ beryllium sulfate suggest that $\mathrm{Be}^{2+}$ ions escape lysosomes and are present in both the cytosol and the nucleus (72), raising the possibility that the ions could affect multiple processes or ion channels inside the cells once they destabilize the lysosomal membranes. Further study is needed to understand the mechanism by which lysosomal leakage leads to loss in membrane integrity of Be-exposed AMs.

We found that Be-induced release of IL-1 $\alpha$ and DNA into the extracellular space was amplified by TNF- $\alpha$ but that only release of DNA was caspase/cathepsin dependent. Be phagocytosis enhanced TNF- $\alpha$ secretion within 2 hours of exposure. TNF- $\alpha$ is known to enhance intracellular levels of IL-1 $\alpha$ in macrophages and other cells via activation of NF- $\kappa B$ by membrane-bound TNFR (complex I) (73, 74). Our kinetic data suggest that these effects occur before 2 hours in Be-exposed AMs, leading to an increase in intracellular IL-1 $\alpha$ stores that were maintained inside the cells until cell death. Our data show that macrophages exposed to Be for more than 2 hours had initiated apoptosis; this was associated with increased caspase- 8 activity and fragmentation of DNA. TNFR-dependent caspase- 8 activation is well known to be mediated by internalized TNFR signaling (via complex II) when NF-kB levels drop (74); however, additional study is need to clarify the relative roles and kinetics of TNFR1 and TNFR2 signaling in Be-exposed AMs. It is possible that a gradual shift between these 2 TNF- $\alpha$ signaling pathways enhanced intracellular levels of both IL-1 $\alpha$ and fragmented DNA following Be phagocytosis in AMs. Be-induced necrosis of the cells that followed these events between 12 and 18 hours resulted in release of both nucleosomal DNA and IL-1 $\alpha$ from the cells, a spectrum of DAMPs that differed from primary necrotic (HK or FT) or necroptotic AMs. In addition to DNA, histones and other nuclear proteins in fragmented chromatin possess DAMP activity $(75,76)$ and could augment the effects of DNA, which together with IL- $1 \alpha$ have potent, redundant effects on promoting upregulation of costimulatory molecule expression on $\mathrm{cDCs}$ and their migration to the LDLNs (2).

The data in this study suggest that the timing of TNF- $\alpha$ release, initiation of apoptosis, and kinetics of plasma membrane disruption affect DAMP release from AMs. These events affected both innate and adaptive arms of the immune system, exacerbating pathogenic T cell responses in a mouse model of CBD. Patients with $\mathrm{CBD}$ who produce higher levels of TNF- $\alpha$ are at risk for higher disease severity, indicating that TNF- $\alpha$ plays an important role in CBD pathogenesis $(77,78)$. A small study on the effects of anti-TNF- $\alpha$ (infliximab) therapy in $\mathrm{CBD}$ patients found that $\mathrm{CD} 4^{+} \mathrm{T}$ cell numbers in the blood and BAL were reduced after treatment and that the remaining T cells expressed lower levels of effector cytokines (IFN- $\gamma$ and TNF- $\alpha$ ). Although the numbers of patients in this study limited the statistical power, 4 out of 5 patients had reduced $\mathrm{Be}^{2+} /$ self-peptide-specific cytokine production $(77,78)$. Thus, in addition to a role in breaking tolerance, TNF- $\alpha$-dependent DAMP release could affect the maintenance of pathogenic T cells in the chronic stages of disease.

TNF- $\alpha$ is associated with the pathogenesis of multiple types of T cell-mediated autoimmune diseases, hypersensitivity, granulomatous inflammation, and pulmonary fibrosis $(79,80)$. It can be made by multiple cell types in the lung and can affect innate and adaptive immune cells as well as tissue epithelial cells, 
endothelial cells, and fibroblasts. In addition, pulmonary exposure to a variety of particles has been linked to development of allergy, asthma, and autoimmune diseases (13-23). Here we show a novel mechanism for TNF- $\alpha$ in regulating AM DAMP release following particle-induced cell death that may underlie the known roles of lung exposures and TNF- $\alpha$ in the development and exacerbation of T cell-mediated autoimmune disease and hypersensitivity.

\section{Methods}

A detailed description of mice, reagents, antibodies, tissue processing, cell lines, tissue culture, and methods for the Supplemental Figures is included in Supplemental Methods.

Mouse treatments. For i.t. instillations, mice were removed from isoflurane (Piramal Critical Care, 803250) anesthesia and supported on a table at $45^{\circ}$. While depressing the tongue, $50 \mu \mathrm{L}$ of Dulbecco's PBS (DPBS) with or without $\mathrm{Be}$ or $\mathrm{BeO}$ was administered into the tracheal opening and observed for aspiration during inhalation. In some experiments, mice were injected i.p. with $100 \mu \mathrm{L}$ of DPBS containing either rat IgG1 isotype control or anti-mouse TNF- $\alpha \mathrm{mAb}$. For generation of bone marrow chimeras, recipient mice were irradiated with 2 doses of 6 Gy separated by a 4-hour break and received approximately $2 \times 10^{6}$ to $5 \times 10^{6}$ bone marrow cells by retro-orbital injection. Mice were maintained on Baytril (AlfaAesar, J60023) water for 4 weeks, and reconstitution was checked by analysis of blood cells 6 to 8 weeks after bone marrow transfer.

Primary tissue collection and processing. BAL was performed with 3 washes of $1 \mathrm{~mL}$ DPBS. LNs and lungs were harvested and processed into single-cell suspensions (see Supplemental Methods). In some experiments, lungs were inflated with 3\% low melt agarose, fixed in phosphate-buffered formalin, and processed for H\&E staining as previously described (26). For quantification of intracellular IL-1 $\alpha$, AMs were subjected to 5 freeze thaw cycles in DPBS containing Halt Protease Inhibitor Cocktail (Thermo Fisher Scientific). Viable cells were counted on a hemocytometer using trypan blue or by flow cytometry with AccuCheck counting beads (Thermo Fisher Scientific) as described in Flow cytometry. Lungs or LN cells were assessed by ELISPOT as previously described (26).

In vitro analysis of primary AMs. One hundred thousand AMs (pooled from BAL of B6 mice) were added to wells of flat-bottomed, 96-well plates $(200 \mu \mathrm{L} /$ well) and allowed to adhere for 30 minutes at $37^{\circ} \mathrm{C}, 10 \% \mathrm{CO}_{2}$, in complete DMEM (cDMEM, described in Supplemental Methods). Nonadherent cells were washed away (confirmed through the microscope). In some experiments, AMs were pretreated with inhibitors or vehicle (DMSO) controls as indicated in figure legends before addition of stimuli. In other experiments, isotype control or TNFR1- or TNFR2-blocking antibodies in cDMEM with 0.01\% DMSO were added for 1 hour before addition of the stimuli. Stimuli were added as indicated in legends. After culture, cells were pelleted at $400 \mathrm{~g}$ for 5 minutes, and SNs were harvested. Particles and debris were removed from SNs by centrifuging at $2000 \mathrm{~g}$ for 30 minutes. Cell-free SNs were tested for dsDNA and cytokines as described in Supplemental Methods.

Ex vivo analysis of extracellular DNA and cytokines in the BALF. Cells were pelleted by centrifuging BAL samples at $400 \mathrm{~g}$ for 5 minutes and analyzed by flow cytometry as described below. BALF was separated from the cells and tested for the presence of cytokines and DNA as described in Supplemental Methods.

Analysis of intracellular IL-1 $\alpha$ in AMs. BAL cells were pooled from untreated mice or from mice exposed i.t. for 2 hours with Be. Viable AMs were quantified by flow cytometry as described below. Cells were pelleted and IL-1 $\alpha$ was quantified in the cell lysates as described in Supplemental Methods. The data were adjusted using original lysate volumes and then normalized to total AM numbers for each sample and expressed as picograms IL-1 $\alpha / 100,000$ AMs.

Flow cytometry. AMs, BAL cells, and LDLN cells were analyzed by flow cytometry as described previously $(2,11,12)$ and in Supplemental Methods. Cells were stained and washed with flow wash comprising DPBS containing 1\% BSA (MilliporeSigma, A2153) and 0.1\% $\mathrm{NaN}_{3}$ (MilliporeSigma, SX0299). In some experiments, cells were washed and stained with Annexin V APC for 15 minutes in Binding Buffer for Annexin V (Invitrogen, Thermo Fisher Scientific, BMS500BB). Immediately before analysis, $2 \times 10^{4} \mathrm{AccuCheck}$ counting beads were added to each sample. Cells and beads were analyzed on a FACSCanto II flow cytometer (BD Biosciences) using FlowJo software (Tree Star). Bead and cell counts for each cell population were determined and total cells per sample calculated by multiplying the ratio of the (cell count/bead count) $\times$ total beads/well $\left(2 \times 10^{4}\right) \times$ dilution factor (L sample stained/ $\mu \mathrm{L}$ total sample).

Statistics. A 1-way ANOVA was used to test for differences for normally distributed data between more than 2 experimental groups, and a 2-way unpaired $t$ test was used to test for differences for normally 
distributed data between 2 groups as noted in the figure legends. A Shapiro-Wilk normality test was used to test for normal distribution. Medians were used to indicate central tendency for data without a normal distribution and means for data with a normal distribution. A Kruskal-Wallis test was used to test for differences between multiple groups. A $P$ value less than 0.05 was considered statistically significant. Data were analyzed using GraphPad Prism.

Study approval. Mice were housed, bred, genotyped, and treated in the Office of Laboratory Animal Resources (OLAR) vivarium in accordance with IACUC-approved protocol standards at the University of Colorado Anschutz Medical Campus.

\section{Author contributions}

MKC and AMS contributed equally in generating the experimental data presented in this manuscript and in the writing of the manuscript. MKC, AMS, MFW, and SMA performed experiments, contributed to design of experiments, analyzed and interpreted the data, and contributed to drafting and editing the manuscript. DKR and MTL performed experiments and analyzed and interpreted data. AKM performed ELISPOTs and mouse genotyping. DGM and SMA assisted with bone marrow chimera experiments and mouse treatment. APF assisted with editing of the manuscript and interpretation of the data. ASM obtained funding for, designed, and oversaw the study; performed experiments; analyzed and interpreted data; and drafted the manuscript.

\section{Acknowledgments}

The authors would like to acknowledge the OLAR vivarium, the ClinImmune Flow Core Facility, Radu Moldovan and the Advanced Light Microscopy Core Facility, the Pathology Core Facility, the Biorepository Core Facility, and the Web-Waring Summer Research Program, all at the University of Colorado Anschutz Medical Campus. We acknowledge Jen Matsuda and the staff in the Mouse Genetics Core Facility at National Jewish Health for generation and characterization of the $M y d 88^{-1-} \mathrm{DP} 2 \mathrm{mice}$. APF is funded by NIH grants ES025534, HL62410, and HL102245. This study was funded by NIH grant number HL126736 to ASM.

Address correspondence to: Amy S. McKee, Box B-164, 12700 East 19th Avenue, Aurora, Colorado 80045, USA. Phone: 303.724.7214; Email: amy.mckee@cuanschutz.edu.

1. Kuroda E, et al. Inhaled fine particles induce alveolar macrophage death and interleukin-1 $\alpha$ release to promote inducible bronchus-associated lymphoid tissue formation. Immunity. 2016;45(6):1299-1310.

2. Wade MF, et al. TLR9 and IL-1R1 promote mobilization of pulmonary dendritic cells during beryllium sensitization. $J$ Immunol. 2018;201(8):2232-2243.

3. Marichal T, et al. DNA released from dying host cells mediates aluminum adjuvant activity. Nat Med. 2011;17(8):996-1002.

4. Yu Q, Wang H, Peng Q, Li Y, Liu Z, Li M. Different toxicity of anatase and rutile $\mathrm{TiO}_{2}$ nanoparticles on macrophages: involvement of difference in affinity to proteins and phospholipids. J Hazard Mater. 2017;335:125-134.

5. Joshi GN, Knecht DA. Silica phagocytosis causes apoptosis and necrosis by different temporal and molecular pathways in alveolar macrophages. Apoptosis. 2013;18(3):271-285.

6. Hamilton RF, Thakur SA, Holian A. Silica binding and toxicity in alveolar macrophages. Free Radic Biol Med. 2008;44(7):1246-1258.

7. Rabolli V, et al. The alarmin IL-1 $\alpha$ is a master cytokine in acute lung inflammation induced by silica micro- and nanoparticles. Part Fibre Toxicol. 2014;11:69.

8. Behbehani K, Beller DI, Unanue ER. The effects of beryllium and other adjuvants on Ia expression by macrophages. J Immunol. 1985;134(4):2047-2049.

9. Hall JG. Studies on the adjuvant action of beryllium. I. Effects on individual lymph nodes. Immunology. 1984;53(1):105-113.

10. Hall JG, Spencer J. Studies on the adjuvant action of beryllium. II. Systemic effects with particular reference to secretory immunity. Immunology. 1984;53(1):115-120.

11. Atif SM, et al. Protective role of B cells in sterile particulate-induced lung injury. JCI Insight. 2019;5:125494.

12. McKee AS, Mack DG, Crawford F, Fontenot AP. MyD88 dependence of beryllium-induced dendritic cell trafficking and CD4 ${ }^{+}$ T-cell priming. Mucosal Immunol. 2015;8(6):1237-1247.

13. Chiu S, et al. Lung injury combined with loss of regulatory T cells leads to de novo lung-restricted autoimmunity. J Immunol. 2016;197(1):51-57.

14. Mikuls TR, Payne JB, Deane KD, Thiele GM. Autoimmunity of the lung and oral mucosa in a multisystem inflammatory disease: the spark that lights the fire in rheumatoid arthritis? J Allergy Clin Immunol. 2016;137(1):28-34.

15. Willemze A, et al. The interaction between HLA shared epitope alleles and smoking and its contribution to autoimmunity against several citrullinated antigens. Arthritis Rheum. 2011;63(7):1823-1832.

16. Li N, et al. A work group report on ultrafine particles (American Academy of Allergy, Asthma \& Immunology): why ambient ultrafine and engineered nanoparticles should receive special attention for possible adverse health outcomes in human subjects. J Allergy Clin Immunol. 2016;138(2):386-396.

17. Toda T, Yoshino S. Amorphous nanosilica particles block induction of oral tolerance in mice. J Immunotoxicol. 2016;13(5):723-728. 
18. Larsson P, et al. Exhaled particles as markers of small airway inflammation in subjects with asthma. Clin Physiol Funct Imaging. 2017;37(5):489-497.

19. Brandt EB, et al. Exposure to allergen and diesel exhaust particles potentiates secondary allergen-specific memory responses, promoting asthma susceptibility. J Allergy Clin Immunol. 2015;136(2):295-303.e7.

20. Alberg T, Hansen JS, Lovik M, Nygaard UC. Particles influence allergic responses in mice--role of gender and particle size. J Toxicol Environ Health Part A. 2014;77(5):281-292.

21. Bernatsky S, Fournier M, Pineau CA, Clarke AE, Vinet E, Smargiassi A. Associations between ambient fine particulate levels and disease activity in patients with systemic lupus erythematosus (SLE). Environ Health Perspect. 2011;119(1):45-49.

22. Sakai M, et al. Diesel exhaust (DE) aggravates pathology of delayed-type hypersensitivity (DTH) induced by methyl-bovine serum albumin (mBSA) in mice. J Toxicol Sci. 2009;34(5):483-492.

23. Ryan $\mathrm{PH}$, et al. Exposure to traffic-related particles and endotoxin during infancy is associated with wheezing at age 3 years. Am J Respir Crit Care Med. 2009;180(11):1068-1075.

24. Clayton GM, et al. Structural basis of chronic beryllium disease: linking allergic hypersensitivity and autoimmunity. Cell. 2014;158(1):132-142.

25. Falta MT, et al. Identification of beryllium-dependent peptides recognized by CD4+ T cells in chronic beryllium disease. $J$ Exp Med. 2013;210(7):1403-1418.

26. Mack DG, et al. Regulatory T cells modulate granulomatous inflammation in an HLA-DP2 transgenic murine model of beryllium-induced disease. Proc Natl Acad Sci U S A. 2014;111(23):8553-8558.

27. Sawyer RT, Maier LA. Chronic beryllium disease: an updated model interaction between innate and acquired immunity. Biometals. 2011;24(1):1-17.

28. Li L, et al. Beryllium increases the CD14(dim)CD16+ subset in the lung of chronic beryllium disease. PLoS ONE. 2015;10(2):e0117276.

29. Steinman RM, Nussenzweig MC. Avoiding horror autotoxicus: the importance of dendritic cells in peripheral T cell tolerance. Proc Natl Acad Sci U S A. 2002;99(1):351-358.

30. Hawiger D, et al. Dendritic cells induce peripheral T cell unresponsiveness under steady state conditions in vivo. $J$ Exp Med. 2001;194(6):769-779.

31. Doan T, McNally A, Thomas R, Steptoe RJ. Steady-state dendritic cells continuously inactivate T cells that escape thymic negative selection. Immunol Cell Biol. 2009;87(8):615-622.

32. Nace G, Evankovich J, Eid R, Tsung A. Dendritic cells and damage-associated molecular patterns: endogenous danger signals linking innate and adaptive immunity. J Innate Immun. 2012;4(1):6-15.

33. Bakdash G, Sittig SP, van Dijk T, Figdor CG, de Vries IJ. The nature of activatory and tolerogenic dendritic cell-derived signal II. Front Immunol. 2013;4:53.

34. Sokolovska A, Hem SL, HogenEsch H. Activation of dendritic cells and induction of CD4(+) T cell differentiation by aluminum-containing adjuvants. Vaccine. 2007;25(23):4575-4585.

35. Liwski RS, Chase JC, Baldridge WH, Sadek I, Rowden G, West KA. Prolonged costimulation is required for naive T cell activation. Immunol Lett. 2006;106(2):135-143.

36. McKee AS, Fontenot AP. Interplay of innate and adaptive immunity in metal-induced hypersensitivity. Curr Opin Immunol. 2016;42:25-30.

37. Yoon S, et al. Caspase-dependent cell death-associated release of nucleosome and damage-associated molecular patterns. Cell Death Dis. 2014;5:e1494.

38. He S, Liang Y, Shao F, Wang X. Toll-like receptors activate programmed necrosis in macrophages through a receptor-interacting kinase-3-mediated pathway. Proc Natl Acad Sci U S A. 2011;108(50):20054-20059.

39. Du C, Fang M, Li Y, Li L, Wang X. Smac, a mitochondrial protein that promotes cytochrome c-dependent caspase activation by eliminating IAP inhibition. Cell. 2000;102(1):33-42.

40. Akara-Amornthum P, Lomphithak T, Choksi S, Tohtong R, Jitkaew S. Key necroptotic proteins are required for Smac mimeticmediated sensitization of cholangiocarcinoma cells to TNF- $\alpha$ and chemotherapeutic gemcitabine-induced necroptosis. $P L o S$ ONE. 2020;15(1):e0227454.

41. Laukens B, et al. Smac mimetic bypasses apoptosis resistance in FADD- or caspase-8-deficient cells by priming for tumor necrosis factor $\alpha$-induced necroptosis. Neoplasia. 2011;13(10):971-979.

42. Sun H, et al. Design, synthesis, and evaluation of a potent, cell-permeable, conformationally constrained second mitochondria derived activator of caspase (Smac) mimetic. J Med Chem. 2006;49(26):7916-7920.

43. Bronner DN, O'Riordan MX. Measurement of mitochondrial DNA release in response to ER stress. Bio Protoc. 2016;6(12):e1839.

44. Carlsen TG, et al. Interleukin-1 $\alpha$ activation and localization in lipopolysaccharide-stimulated human monocytes and macrophages. J Immunol Methods. 2015;422:59-71.

45. Kim B, et al. The interleukin-1 $\alpha$ precursor is biologically active and is likely a key alarmin in the IL-1 family of cytokines. Front Immunol. 2013;4:391.

46. Gibbings SL, et al. Transcriptome analysis highlights the conserved difference between embryonic and postnatal-derived alveolar macrophages. Blood. 2015;126(11):1357-1366.

47. Dixon SJ, et al. Ferroptosis: an iron-dependent form of nonapoptotic cell death. Cell. 2012;149(5):1060-1072.

48. Hornung V, et al. Silica crystals and aluminum salts activate the NALP3 inflammasome through phagosomal destabilization. Nat Immunol. 2008;9(8):847-856.

49. Joshi GN, Goetjen AM, Knecht DA. Silica particles cause NADPH oxidase-independent ROS generation and transient phagolysosomal leakage. Mol Biol Cell. 2015;26(18):3150-3164.

50. Kittle LA, Sawyer RT, Fadok VA, Maier LA, Newman LS. Beryllium induces apoptosis in human lung macrophages. Sarcoidosis Vasc Diffuse Lung Dis. 2002;19(2):101-113.

51. Sawyer RT, Fadok VA, Kittle LA, Maier LA, Newman LS. Beryllium-stimulated apoptosis in macrophage cell lines. Toxicology. 2000;149(2-3):129-142. 
52. Grivennikov SI, Kuprash DV, Liu ZG, Nedospasov SA. Intracellular signals and events activated by cytokines of the tumor necrosis factor superfamily: From simple paradigms to complex mechanisms. Int Rev Cytol. 2006;252:129-161.

53. Gozal E, Ortiz LA, Zou X, Burow ME, Lasky JA, Friedman M. Silica-induced apoptosis in murine macrophage: involvement of tumor necrosis factor-alpha and nuclear factor-kappaB activation. Am J Respir Cell Mol Biol. 2002;27(1):91-98.

54. Meredith MM, et al. Expression of the zinc finger transcription factor zDC (Zbtb46, Btbd4) defines the classical dendritic cell lineage. J Exp Med. 2012;209(6):1153-1165.

55. Satpathy AT, et al. Zbtb46 expression distinguishes classical dendritic cells and their committed progenitors from other immune lineages. J Exp Med. 2012;209(6):1135-1152.

56. Mulay SR, et al. Cytotoxicity of crystals involves RIPK3-MLKL-mediated necroptosis. Nat Commun. 2016;7:10274

57. Radloff M, Delling M, Gercken G. Protein phosphorylation in alveolar macrophages after stimulation with heavy metal-coated silica particles. Toxicol Lett. 1998;96-97:69-75.

58. Rabolli V, Thomassen LC, Uwambayinema F, Martens JA, Lison D. The cytotoxic activity of amorphous silica nanoparticles is mainly influenced by surface area and not by aggregation. Toxicol Lett. 2011;206(2):197-203.

59. Napierska D, et al. Size-dependent cytotoxicity of monodisperse silica nanoparticles in human endothelial cells. Small. 2009;5(7):846-853.

60. Dubois CM, Bissonnette E, Rola-Pleszczynski M. Asbestos fibers and silica particles stimulate rat alveolar macrophages to release tumor necrosis factor. Autoregulatory role of leukotriene B4. Am Rev Respir Dis. 1989;139(5):1257-1264.

61. Iyer R, Hamilton RF, Li L, Holian A. Silica-induced apoptosis mediated via scavenger receptor in human alveolar macrophages Toxicol Appl Pharmacol. 1996;141(1):84-92.

62. Iyer R, Holian A. Involvement of the ICE family of proteases in silica-induced apoptosis in human alveolar macrophages. Am J Physiol. 1997;273(4):L760-L767.

63. Kim JK, et al. Mechanism of silica- and titanium dioxide-induced cytotoxicity in alveolar macrophages. J Toxicol Environ Health Part A. 1999;58(7):437-450.

64. Zhang Z, Shen HM, Zhang QF, Ong CN. Involvement of oxidative stress in crystalline silica-induced cytotoxicity and genotoxicity in rat alveolar macrophages. Environ Res. 2000;82(3):245-252.

65. Thibodeau M, Giardina C, Hubbard AK. Silica-induced caspase activation in mouse alveolar macrophages is dependent upon mitochondrial integrity and aspartic proteolysis. Toxicol Sci. 2003;76(1):91-101.

66. Thibodeau MS, Giardina C, Knecht DA, Helble J, Hubbard AK. Silica-induced apoptosis in mouse alveolar macrophages is initiated by lysosomal enzyme activity. Toxicol Sci. 2004;80(1):34-48.

67. Kool M, et al. Cutting edge: alum adjuvant stimulates inflammatory dendritic cells through activation of the NALP3 inflammasome. J Immunol. 2008;181(6):3755-3759.

68. Paladini F, et al. Divergent effect of cobalt and beryllium salts on the fate of peripheral blood monocytes and T lymphocytes Toxicol Sci. 2011;119(2):257-269.

69. Sawyer RT, et al. Beryllium-stimulated reactive oxygen species and macrophage apoptosis. Free Radic Biol Med. 2005;38(7):928-937.

70. Day GA, et al. Bioavailability of beryllium oxide particles: an in vitro study in the murine J774A.1 macrophage cell line model. Exp Lung Res. 2005;31(3):341-360.

71. Price DJ, Joshi JG. Ferritin. Binding of beryllium and other divalent metal ions. J Biol Chem. 1983;258(18):10873-10880.

72. Witschi HP, Aldridge WN. Uptake, distribution and binding of beryllium to organelles of the rat liver cell. Biochem J. 1968;106(4):811-820.

73. Portillo G, Turner M, Chantry D, Feldmann M. Effect of cytokines on HLA-DR and IL-1 production by a monocytic tumour, THP-1. Immunology. 1989;66(2):170-175.

74. Micheau O, Tschopp J. Induction of TNF receptor I-mediated apoptosis via two sequential signaling complexes. Cell. 2003;114(2):181-190.

75. Magna M, Pisetsky DS. The alarmin properties of DNA and DNA-associated nuclear proteins. Clin Ther. 2016;38(5):1029-1041.

76. Huang H, et al. Endogenous histones function as alarmins in sterile inflammatory liver injury through Toll-like receptor 9 in mice. Hepatology. 2011;54(3):999-1008.

77. Maier LA, et al. Infliximab therapy modulates an antigen-specific immune response in chronic beryllium disease. Respir Med. 2012;106(12):1810-1813.

78. Maier LA, et al. High beryllium-stimulated TNF-alpha is associated with the $-308 \mathrm{TNF}$-alpha promoter polymorphism and with clinical severity in chronic beryllium disease. Am J Respir Crit Care Med. 2001;164(7):1192-1199.

79. Hou J, et al. TNF- $\alpha$-induced NF- $\mathrm{kB}$ activation promotes myofibroblast differentiation of LR-MSCs and exacerbates bleomycin-induced pulmonary fibrosis. J Cell Physiol. 2018;233(3):2409-2419.

80. Atzeni F, Turiel M, Capsoni F, Doria A, Meroni P, Sarzi-Puttini P. Autoimmunity and anti-TNF-alpha agents. Ann N Y Acad Sci. 2005;1051:559-569. 